Chapter 11

\title{
Applications of \\ Confocal Laser Scanning Microscopy (CLSM) in Foods
}

\author{
Jaime A. Rincón Cardona, Cristián Huck Iriart and \\ María Lidia Herrera
}

Additional information is available at the end of the chapter

http://dx.doi.org/10.5772/55653

\section{Introduction}

Much of the work in the area of physical properties of fats is aimed at determining the relationship among triglyceride structure, crystal properties, crystallization conditions, and macroscopic properties of fats. In finished product containing fat, some of these many macroscopic properties include spredability of margarine, butter and spreads; snap of chocolate; blooming of chocolate; and graininess, smoothness, mouthfeel, water binding, and emulsion stability of spreads [1]. Plastic fats consist of a crystal network in a continuous oil matrix. Many articles in the past have been focused on establishing relationships between lipid composition or polymorphism and macroscopic properties of fats without much consideration of the microstructure of the fat crystal network. Germane to a thorough understanding of plastic fat rheology is a characterization of its microstructure. Not including microstructure as a variable will lead to failure in the prediction of macroscopic properties. In many other non fat or low fat products macroscopic properties depend on their structural organization. Emulsion stability, which is one of the most important physical properties of multiple-phase systems, is strongly determined by oil droplet size and interactions among components that determine spatial distribution of lipid and aqueous phases. Thus, control of food properties for various applications requires a better understanding of the relationships between the food microstructure and macroscopic properties.

Light microscopy is a well-developed and increasingly used technique for studying the microstructure of food systems in relation to their physical properties and processing behavior. To obtain good-quality, high-resolution images of the internal structures of foods it is necessary to cut thin sections of the sample. Procedures that applied substantial shear and compressive forces may destroy or damage structural elements, and sectioning is time consuming and involves chemical processing steps that may introduce artifacts and make image 
interpretation difficult. Investigations of microstructural changes in foods are increasingly common especially with the growing availability of new microscopic techniques such as the confocal laser scanning microscopy (CLSM) that can probe in situ changes in the microstructure without disturbing the sample. In this instrument, image formation does not depend on transmitting light through the specimen, and, therefore, bulk speciments can be used for the first time in light microscopy. Thus, the CLSM does not require sample fixation and/or dehydration. In addition, when combined with three-dimensional (3-D) reconstruction techniques, optical sectioning may be sufficient to reveal novel information typically unobtainable via traditional two-dimensional (2-D) micrographs since in CLSM information from regions distant from the plane of focus does not blur the image of the focal plane [2]. The primary value of the CLSM to research is its ability to produce optical sections through a three-dimensional (3-D) specimen, for example a thick object such as cheese, yogurt or chocolate. The instrument uses a focused scanning laser to illuminate a subsurface layer of the specimen in such a way that information from this focal plane passes back through the specimen and is projected onto a pinhole (confocal aperture) in front of a detector. Only a focal plane image is produced, which is an optical slice of the structure at some preselected depth within the sample. By moving the specimen up and down relative to the focused laser light, a large number of consecutive optical sections with improved lateral resolution (compared with conventional light microscopy) can be obtained with a minimum of sample preparation. A further advantage of CLSM is the possibility to follow in situ the dynamics of processes such as phase separation, coalescence, aggregation, coagulation, solubilization, etc. Specially designed stages, which allow heating, cooling or mixing of the sample, give the possibility to simulate food processing under the microscope [3].

CLSM has been used in food science since the eighties. Several reviews discussing the application of this technique in microstructural studies of food products have been reported in literature $[1,4,5]$. These reviews have shown the advantages of using CLSM over conventional techniques for studying the relation between the composition, processing, and final properties of food products [6]. The optical sectioning capability of CLSM has proved very useful in the examination of high-fat foods, which are difficult to prepare using the conventional microscopy without the loss or migration of fat globules [7]. In systems like cheese, one of the advantages of this technique is that it can both visualize and chemically differentiate cheese components through the use of protein and lipid specific stains. As internal probes cannot be excited using the lasers typically installed on commercial confocal microscopes, extrinsic fluorechromes must be used such as acridine orange, Nile blue, fluorescein isothiocyanate (FITC), rhodamine B and fast green FCF to stain protein and Nile red to stain fat [8]. An even more detail analysis of food structure may be achieved by the simultaneous labeling of two or more components of foods with probes which are specific for each component. In applications of CLSM in food science there is a minimum sample preparation. A detailed protocol which described how to stain a fat system with Nile red was reported by Herrera and Hartel [6]. Besides, there are no special techniques or modifications to the equipment for food science. The techniques of microscopy are identical as for the life science. The possibility to combine CLSM with rheological measurements, light scattering and other physical analytical techniques in the same experiments with specially designed stages allows obtaining detailed structural information of complex food systems. This chapter reviews applications of CLSM in food systems. Results on bulk fat, emulsions, gels, and a variety of products are reported. 


\section{Description of fats in bulk}

Milk fat has a unique taste and is therefore an important ingredient in many food products. The composition of milk fat is complex, e.g., it contains at least 400 different fatty acids of which 12 are present in proportions greater than $1 \%$. Therefore, the diversity of triglycerides species in milk fat is enormous, resulting in broad crystallization and melting ranges. Furthermore, the breed and feeding of the cow have influence on the milk fatty acids composition, which has impact on the crystallization behavior for anhydrous milk fat and cream. The macroscopic properties of a fat are influenced by a hierarchy of factors. The solid-like behavior in particular is influenced by the amount of solid fat present, the type of crystals, and the interactions among crystals leading to the formation of a fat crystal network. Lipid composition and crystallization conditions will influence crystal habit upon crystallization. Thus, different polymorphic forms and crystal morphologies are possible. Crystallization of milk fat affects several properties that are important for product quality, such as texture, mouthfeel, and rheology. Processing factors such as cooling rate, final temperature and agitation rate are very relevant to crystallization behavior and will also affect product quality. Several authors have studied the microstructure and rheology of fractions of milk fat. Herrera and Hartel [9] have used CLSM to describe the microstructure of blends of 30,40, and 50\% high-melting fraction [Mettler dropping point (MDP) $=$ $47.5^{\circ} \mathrm{C}$ ] in the low-melting fraction $\left(\mathrm{MDP}=16.5^{\circ} \mathrm{C}\right.$ ) of milk fat. The effect of cooling and agitation rates, crystallization temperature, chemical composition of the blends, and storage time on crystalline microstructure (number, size, distribution, etc.) was investigated by CLSM. Samples were crystallized at the selected crystallization temperatures and then were stored at $10^{\circ} \mathrm{C} / \mathrm{min}$. Therefore, two levels of structure were found: the primary crystals which were quantified (number and size) by using other type of microscopy (polarized light microscopy, PLM) and the final structure whose distribution was qualitatively described by CLSM. This distribution was very relevant to understand rheological properties. To improve resolution, a mix of Nile blue and Nile red dyes was dissolved in the melted samples in proportions that did not modify the nucleation kinetics. When the high melting fraction of milk fat was studied the effect of processing conditions on microstructure was evident. Figure 1 reports the microstructure obtained when milk fat was crystallized at $0.1^{\circ} \mathrm{C} / \mathrm{min}$ and $5.5^{\circ} \mathrm{C} / \mathrm{min}$. The dark elements are crystals. The liquid oil is bright. Slowly crystallized samples $\left(0.2^{\circ} \mathrm{C} / \mathrm{min}\right)$ formed different structures from rapidly crystallized samples $\left(5.5^{\circ} \mathrm{C} / \mathrm{min}\right)$. When slow cooling was used, crystals were sometimes diffuse and hard to distinguish from the liquid. Samples were darker as a result of this solid-mass distribution. However, rapidly crystallized samples had well-defined crystals and seemed to be separated by a distinct liquid phase. These crystals were not in touch with each other as was the case for slowly crystallized samples. Figure 1 showed that slow cooling promoted crystal growth. Fewer crystals with bigger size were obtained, the size of which was quantified by PLM. These microstructures are expected to be related to different mechanical properties, being structures with small crystals harder samples than the ones with greater crystals. 

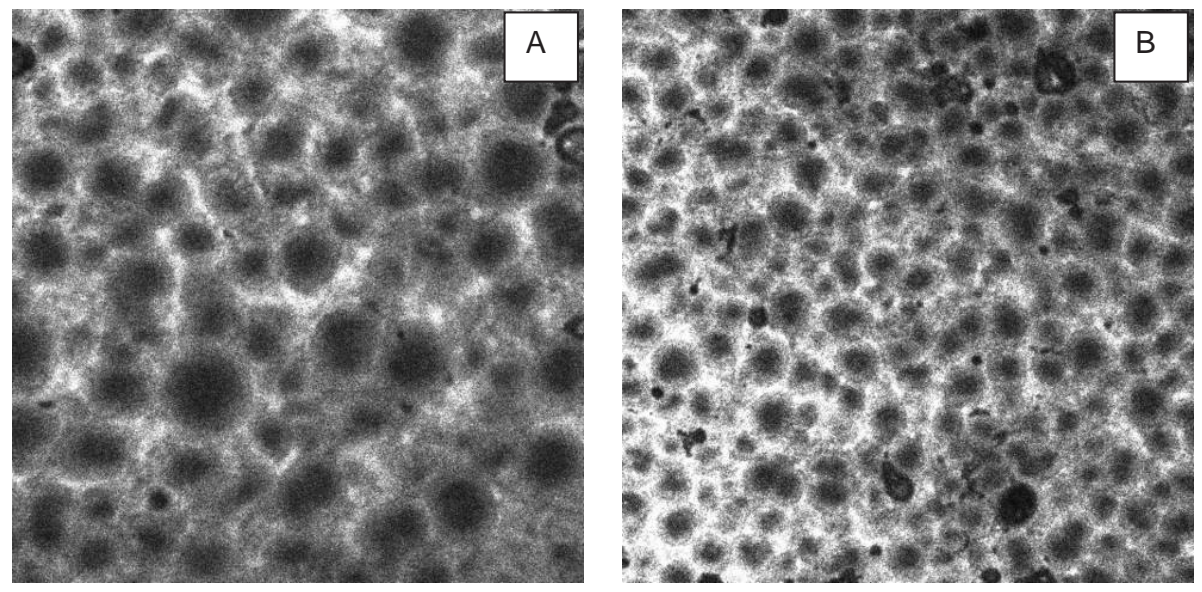

Figure 1. Effect of cooling rate on crystal size for milk fat after $90 \mathrm{~min}$ at $35^{\circ} \mathrm{C}$ and $24 \mathrm{~h}$ at $10^{\circ} \mathrm{C} / \mathrm{min}$. A) slow cooling $\left(0.1^{\circ} \mathrm{C} / \mathrm{min}\right)$ and $\left.\mathrm{B}\right)$ fast cooling $\left(5.5^{\circ} \mathrm{C} / \mathrm{min}\right)$.

When agitation rate was tested, higher agitation rates led to smaller crystal size due to enhanced nucleation. Temperature also had a strong impact in crystal size. Larger crystals were formed when crystallization occurred at higher temperatures. High-melting fraction of Milk fat crystals grew during storage: the longer storage time the greater crystal size. The blends of different fractions (high and low melting) of milk fat showed the same microstructural behavior as high melting fraction of milk fat. Greater crystals were found when samples were crystallized at slow cooling rate, slower agitation rate $(50 \mathrm{rpm})$ and higher temperatures $\left(30^{\circ} \mathrm{C}\right),[9]$.

Wiking et al. [10] performed further studies to investigate the relationship of microstructure and macroscopic properties analyzing non-fractioned anhydrous milk fat. Using differential scanning calorimetry, synchrotron time resolve $X$-ray diffraction and pulsed nuclear magnetic resonance, crystallization mechanisms of milk fat were elucidated. Under the same crystallization conditions, the microstructure of the milk fat was analyzed with CLSM and oscillatory rheology. The milk fat was cooled without agitation to $20^{\circ} \mathrm{C}$ at two different cooling rates, i.e., 0.1 and $10^{\circ} \mathrm{C} / \mathrm{min}$. Thereafter, the isothermal crystallization at $20^{\circ} \mathrm{C}$ was monitored. In agreement with the results shown in Figure 1, faster cooling resulted in a two-step crystallization, and a microstructure that comprised smaller and more uniform crystals than was the case with slower cooling. Consequently, the final texture of the faster cooled milk fat was firmer, i.e., higher complex modulus, than that of the slower cooled milk. X-ray diffraction showed that the two-step crystallization involved a polymorphic transition from $\alpha$ to $\beta^{\prime}$ phase.

Milk fat fractions have found application in a variety of food products. The high melting point stearins are usefulin puffy pastry, whereas the mid fractions are usefulinDanish cookies. Stearins are also used in the reduction in blooming properties of chocolate. The modification by blending of milk fatstearins is an interesting and important approach for utilization of milk fat fractions in a number of edible fat products. Spreadable butter products based on milk fat blended with 
vegetable oils are becoming increasingly popular and a growing market motivates the industry to develop novel products in this category. Martini et al. [11, 12] studied the effect of blending sunflower oil with a high-melting fraction of milk fat using PLM and CLSM. Figure 2 shows two PLM micrographs of a blend of $90 \mathrm{wt}$.\% high-melting fraction of milk fat and $10 \mathrm{wt} . \%$ sunflower oil (A) and $60 \mathrm{wt} . \%$ high-melting fraction of milk fat and $40 \mathrm{wt} . \%$ sunflower oil (B) after $90 \mathrm{~min}$ of crystallization at $40^{\circ} \mathrm{C}$. Samples were crystallized with a cooling rate of $0.1^{\circ} \mathrm{C} / \mathrm{min}$. As may be noticed in Figure 2, the addition of sunflower oil markedly increased crystal size and delayed crystallizationkinetics. Lesscrystals(coming fromasmallernumber of nuclei) wereformed when $40 \mathrm{wt}$.\% sunflower oil was added to high-melting fraction of milk fat. A quantitative analysis of these systems may be found in Martini et al. [11, 12].
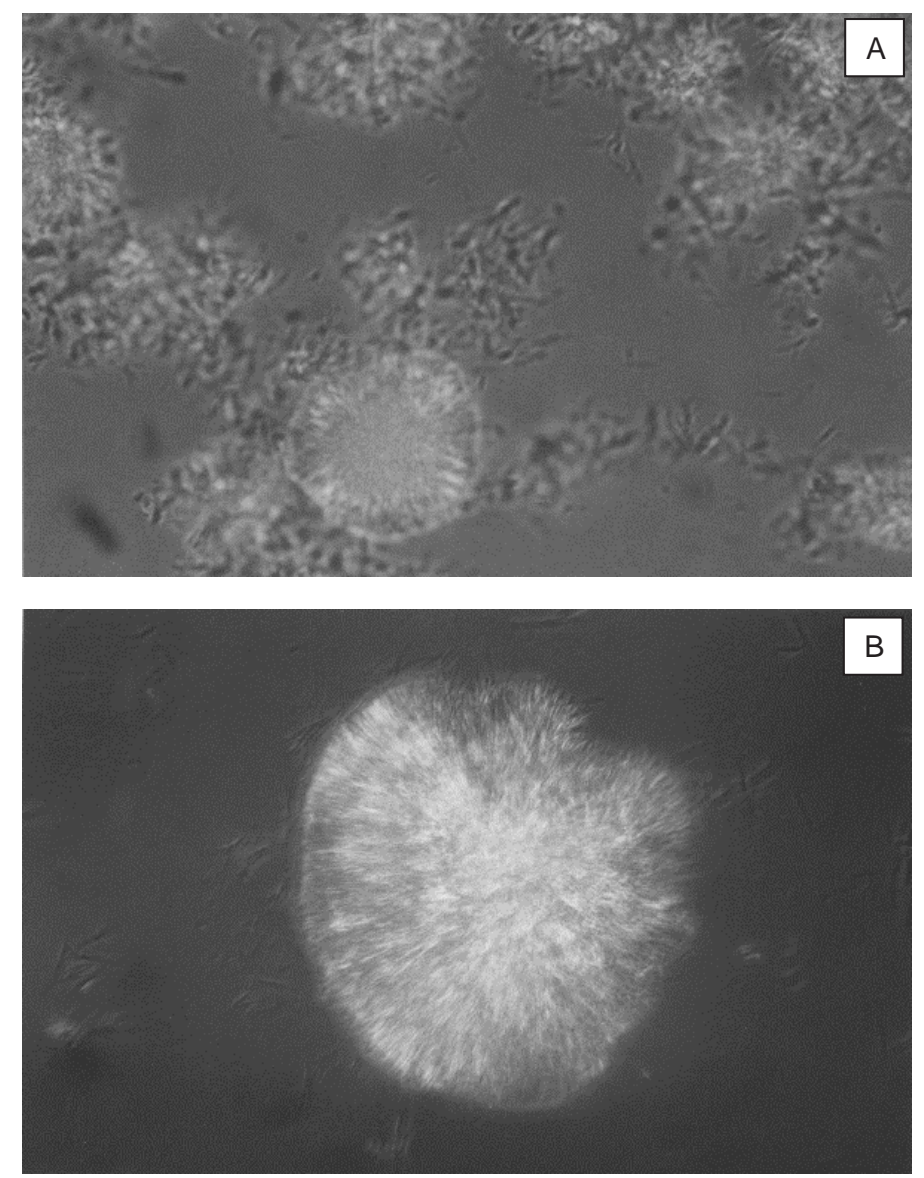

Figure 2. PLM images of two different blends of high-melting fraction of milk fat and sunflower oil crystallized at $40^{\circ} \mathrm{C}$ for 90 min: A) a blend of $90 \mathrm{wt}$ \% high-melting fraction of milk fat and $10 \mathrm{wt}$ \% sunflower oil and B) a blend of $60 \mathrm{wt}$. $\%$ high-melting fraction of milk fat and 40 wt.\% sunflower oil. 
Figure 3 shows CLSM images of two different blends of high-melting fraction of milk fat and sunflower oil: A) a blend of 90 wt.\% high-melting fraction of milk fat and $10 \mathrm{wt} . \%$ sunflower oil and B) a blend of $60 \mathrm{wt} . \%$ high-melting fraction of milk fat and $40 \mathrm{wt} . \%$ sunflower oil. These images correspond to the PLM images report in Figure 2. The blends were crystallized in twosteps: first they were kept at $40^{\circ} \mathrm{C}$ for $90 \mathrm{~min}$ (as in samples in Figure 2) and secondly they were stored at $10^{\circ} \mathrm{C}$ for $24 \mathrm{~h}$. Slow cooling rate $\left(0.1^{\circ} \mathrm{C} / \mathrm{min}\right)$ was used in both cases. In the first step big dark crystals were formed. Then, smaller crystals appeared on the background when samples were cooled to $10^{\circ} \mathrm{C}$. The formed structures were too opaque to study by PLM, therefore they were analyzed by CLSM. The advantage of this approach is that the real microstructure can be described without diluting the system. It may be noticed in Figure 3 that addition of sunflower oil increased primary crystals size. In addition, more separated crystals and a clear liquid phase were formed in the background when blends were stored at $10^{\circ} \mathrm{C}$.
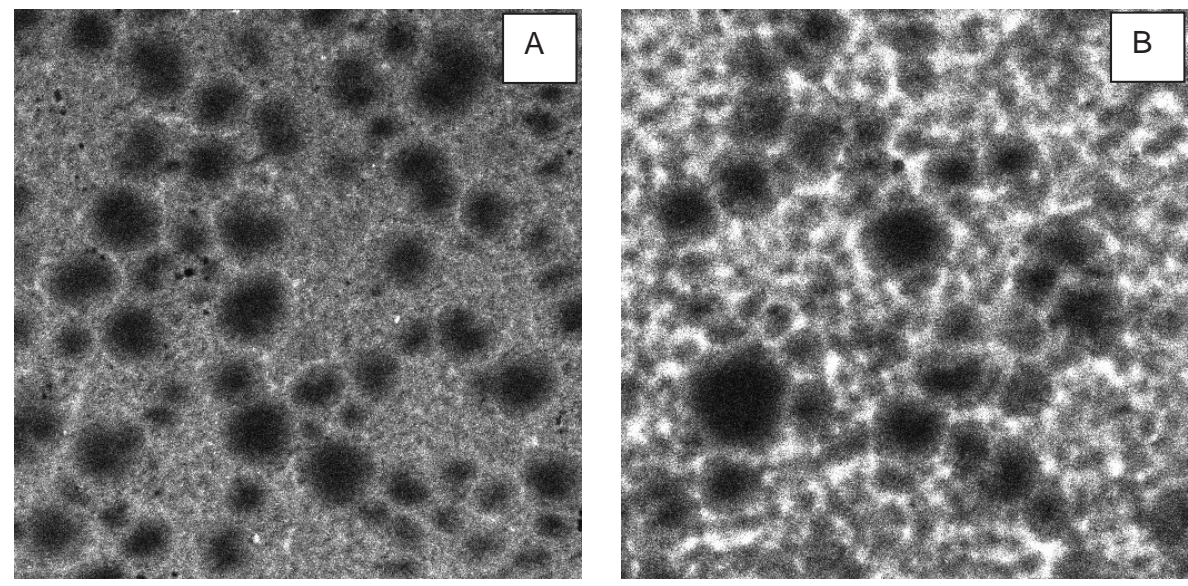

Figure 3. CLSM images of two different blends of high-melting fraction of milk fat and sunflower oil crystallized at $40^{\circ} \mathrm{C}$ for $90 \mathrm{~min}$ and then stored at $10^{\circ} \mathrm{C}$ for $24 \mathrm{~h}$ : A) a blend of $90 \mathrm{wt} . \%$ high-melting fraction of milk fat and $10 \mathrm{wt} . \%$ sunflower oil and B) a blend of 60 wt.\% high-melting fraction of milk fat and $40 \mathrm{wt} . \%$ sunflower oil.

Martini et al. [11, 12] also investigated the effect of cooling rate on microstructure of the blends. Cooling rate is known to affect the polymorphic state of the fat crystals as well as its microstructure and texture. Slow cooling usually promotes stable polymorphic forms such as the $\beta^{\prime}$ or $\beta$ forms while less stable polymorphs are obtained using fast cooling, typically the $\alpha$ form. The $\beta$ form generally leads to greater crystals or harder texture than the other polymorphs. Thus, the different polymorphic forms have different microstructure and different textures. Fast cooling causes rapid development of the thermodynamic driving force for crystallization compared with slow cooling and therefore more and smaller crystals are formed. Figure 4 shows the effect of cooling rate on a $20 \mathrm{wt}$.\% sunflower oil in high-melting fraction of milk fat blend microstructure. The blend was crystallized in two-steps. The first step took place in dynamic conditions with an agitation rate of $50 \mathrm{rpm}$. After $90 \mathrm{~min}$ at $35^{\circ} \mathrm{C}$ samples were cooled 
to $10^{\circ} \mathrm{C}$ and stored in quiescent conditions for $24 \mathrm{~h}$ before CLSM images were taken. Big crystals were obtained in the first step while small crystals were obtained during quiescent crystallization at $10^{\circ} \mathrm{C}$. It may be noticed that when fast cooling was applied, nucleation predominated over crystal growth, resulting in more crystals with smaller crystal size. During fast cooling, triacylglycerols were forced to adopt a crystal structure at conditions far from equilibrium, forming mixed or compound crystals. Fast cooling has been shown to yield a higher solid fat content than slow cooling proving that lower melting point triacylglycerols are included in higher melting point triacylglycerols network. Slow cooling allows triacylglycerols to organize in the right conformation to form crystals and more pure crystals with triacylglycerols of higher melting point are obtained resulting in a lower solid fat content and a higher melting point. In all cases the selected blends crystallized in the same polymorphic form, the $\beta^{\prime}$ form. Although cooling rate had a strong influence in microstructure of high-melting fraction of milk fat/ sunflower oil blends, it did not have much influence in polymorphism of these systems.

Kaufmann et al. [13] studied anhydrous milk fat and its blends with rapeseed oil containing up to $40 \%(\mathrm{w} / \mathrm{w})$ of theoil.Sampleswerecrystallized using aslow $\left(0.05^{\circ} \mathrm{C} / \mathrm{min}\right) \operatorname{orfast}\left(5^{\circ} \mathrm{C} / \mathrm{min}\right)$ cooling rate. Melting behavior was examined using differential scanning calorimetry, texture was analyzed by parallel plate compression tests, microstructure was studied by confocal laser scanning microscopy, and solid fat content was measured by pulse-Nuclear Magnetic Resonance.Inslow cooled samplesaddition of rapeseed oildecreased thehardness, which wasascribed to an increase in crystal cluster size. Similar systems were studied by Buldo and Wiking [14]. In agreement with Kaufmann et al. [13], the microstructure found for the blends of butter and rapeseed oil, as analyzed with CLSM, explained the effect on the rheological behavior. The microstructure analysis showed that a high content of rapeseed oil and high processing temperatures produce a less dense crystal network and a change in protein/water distribution.
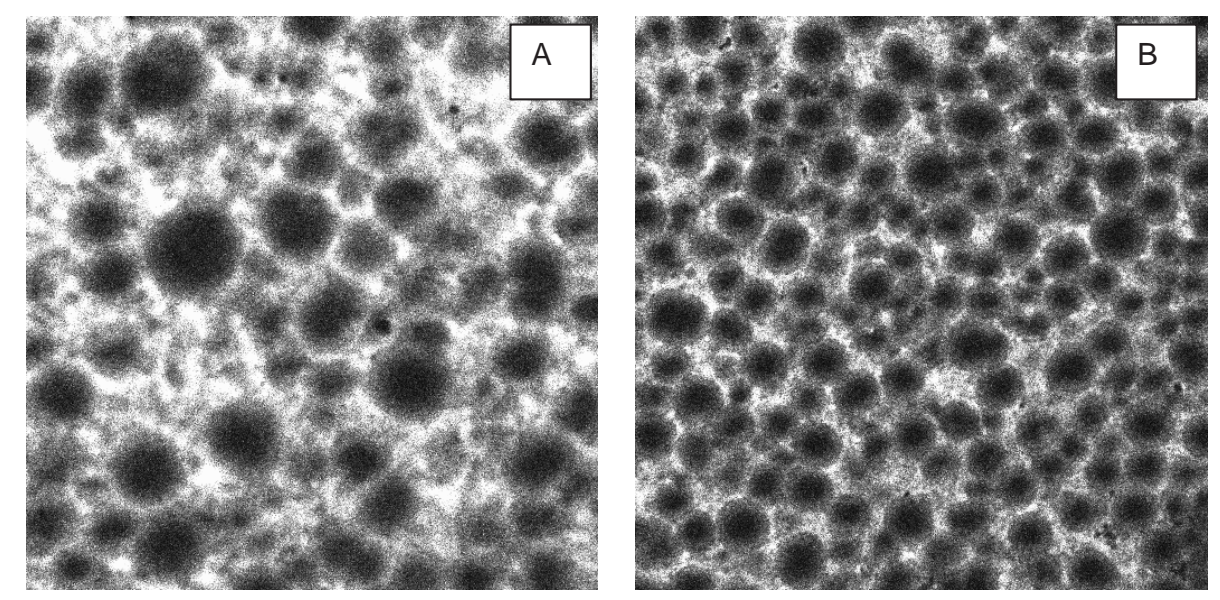

Figure 4. Effect of cooling rate on crystal size for a blend of sunflower oil/high-melting fraction of milk fat after 90 min at $35^{\circ} \mathrm{C}$ and $24 \mathrm{~h}$ at $10^{\circ} \mathrm{C} / \mathrm{min}$. A) slow cooling $\left(0.1^{\circ} \mathrm{C} / \mathrm{min}\right)$ and $\left.\mathrm{B}\right)$ fast cooling $\left(5.5^{\circ} \mathrm{C} / \mathrm{min}\right)$. 


\section{Biopolimer mixtures}

Over the last two decades there has been a resurgence in the study of biopolymer mixtures and in the development of "novel" processing technologies such as High-Pressure Processing. While, the former is motivated by the fact that polysaccharides and proteins almost always coexist in food products, the later is motivated by the need to preserve food products and extend their shelf-life, without affecting their organoleptic and nutritional properties. To explore both aspects, Hemar et al. [15] performed an experimental study on the effect of High Hydrostatic Pressure on the flow behavior of skim milk-gelatin mixtures. The mixtures which contained 0-1 wt.\% gelatin were subjected to different pressures (0-600 MPa) for $15 \mathrm{~min}$ at an initial temperature of $5{ }^{\circ} \mathrm{C}$. The behavior of these mixtures was compared to that of aqueous gelatin solutions subjected to the same pressure treatments. Microstructural observations using confocal scanning laser microscopy, small-deformation oscillatory rheology and particle size measurements were performed in an attempt to relate the microstructural properties of these skim milk-gelatin mixtures to their flow behavior. According to the authors this fundamental work, dealing with the effect of high pressure on the physicochemical properties skim milkgelatin mixtures could be relevant to the industry in several ways. Firstly, skim milk-gelatin mixtures are widely used in the dairy industry, particularly in yoghurt manufacture, where gelatin is used as a stabilizer. In addition the application of High Hydrostatic Pressure to such a system is also relevant, as this technology could be used as a substitute to the conventional heat treatment processes. Secondly, an important finding of this study is that under certain conditions of high pressure and gelatin concentration, an increase in viscosity is observed at intermediate shear-rate (between 10 and $100 \mathrm{~s}-1$ ). This is highly relevant to industry if the system requires subsequent pumping. Thirdly, from a sensory view point, this range of shear rates (10 and $100 \mathrm{~s}-1)$ is comparable to that experienced by a food bolus during swallowing. Thus, this effect of high pressure on the viscosity can influence sensory attribute of the skim milk-gelatin food system.

\section{Emulsion systems}

Oil-in-Water emulsions consist of small lipid droplets dispersed within an aqueous environment. The lipid droplets are normally coated by a thin layer of emulsifier molecules to prevent them from aggregating. In the food industry, a variety of different kinds of surface-active molecules are used as emulsifiers, including small molecule surfactants, phospholipids, proteins, and polysaccharides. Among proteins, sodium caseinate is widely used as an ingredient in the food industry because its functional properties include emulsification, water-binding, fat-binding, thickening, and gelation. It contains a soluble mixture of surface active caseins ( $\alpha s 1_{-}, \alpha s 2-, \beta-$, and $\left.\kappa-\right)$. The caseins adsorb rapidly at the oil-water interface during emulsification and provide long-term stability to oil-in-water emulsions due to a combination of electrostatic and steric stabilization. In milk protein-stabilized emulsions, the presence of ethanol can confer increased stability by reducing the interfacial tension between oil and aqueous phases and so producing a lower average droplet size during 
emulsification. Motivated by separate observations of the sensitivity of emulsion flocculation to $\mathrm{Ca}^{2+}$ content and ethanol concentration, Radford et al. [16] investigated the combined roles of ionic calcium content and ethanol concentration as variables controlling depletioninduced flocculation in caseinate emulsion systems. Construction of the global stability diagram was based on a combination of techniques, including rheology, particle-size distribution analysis, visual creaming observations, and CLSM. The emulsion microstructure at various caseinate-calcium-ethanol compositions has been observed by CLSM using the fluorescent dye Rhodamine B. This dye stains only the protein. On the addition of a moderate concentration of ethanol $(15 \mathrm{wt} \%)$, the dense flocculated protein network appeared to be completely broken down. At that point the microstructure was made up of discrete flocculated droplets separated by relatively large distances. This difference in microstructure with $15 \mathrm{wt}$.\% alcohol addition suggested that alcohol enhance stability at this concentration. A single narrow stable (noncreaming) region was identified, indicating limited cooperation between calcium ions and ethanol.

Physical instability results in an alteration in the spatial distribution or structural organization of the molecules. Creaming, flocculation, coalescence, partial coalescence, phase inversion, and Ostwald ripening are examples of physical instability. The development of an effective strategy to prevent undesirable changes in the properties of a particular food emulsion depends on the dominant physicochemical mechanism(s) responsible for the changes. In practice, two or more of these mechanisms may operate in concert. It is therefore important for food scientists to identify the relative importance of each mechanism, the relationship between them, and the factors that influence them, so that effective means of controlling the stability and physicochemical properties of emulsions can be established. Emulsions have been studied by numerous techniques, such as particle sizing, microscopy, rheology, among others, to characterize their physical properties. Most of these techniques involve some form of dilution. This dilution disrupts some structures that contribute to destabilization. The ability to study the stability of food emulsions in their undiluted forms may reveal subtle nuances about their stability. That is the case of CLSM and another new techniques such as Turbiscan. The Turbiscan method, allows scan the turbidity profile of an emulsion along the height of a glass tube filled with the emulsion, following the fate of the turbidity profile over time. The analysis of the turbidity profiles leads to quantitative data on the stability of the studied emulsions and allows making objective comparisons between different emulsions. Turbiscan measurements together with dynamic light scattering measurements are two techniques that allow quantifying the microstructures described by CLSM. Alvarez Cerimedo et al. [17] studied emulsions stabilized by sodium caseinate. In that article the effect of trehalose on emulsion stability was followed by Turbiscan, the microstructure of emulsions was described by CLSM using Nile red as fluorechrome, and the particle size distribution of emulsion droplets was studied by dynamic light scattering. The fat phase appears in red in CLSM images. The volume-weighted mean diameter $\left(D_{4,3}\right)$, volume percentage of particles exceeding $1 \mu \mathrm{m}$ in diameter $\left(\% V_{\mathrm{d}>1}\right)$, and width of the distribution $(W)$ of emulsions formulated with $10 \mathrm{wt} . \%$ fish oil as lipid phase and different concentrations of trehalose or NaCas are summarized in Table 1. 


\begin{tabular}{ccccc}
\hline \multicolumn{2}{c}{ Sample } & $\begin{array}{c}\boldsymbol{D}_{4,3} \\
(\boldsymbol{\mu m})\end{array}$ & $\begin{array}{c}\boldsymbol{\%} \boldsymbol{V}_{\boldsymbol{d > 1}} \\
(\boldsymbol{\%})\end{array}$ & $\begin{array}{c}\boldsymbol{W} \\
(\boldsymbol{\mu m})\end{array}$ \\
\hline NaCas & $\mathbf{T}$ & 0.49 & 8.22 & 0.85 \\
\hline 4.5 & 0 & 0.37 & 2.71 & 0.52 \\
5.0 & 0 & 0.46 & 7.18 & 0.80 \\
5.5 & 0 & 0.47 & 7.21 & 0.80 \\
6.0 & 0 & 0.46 & 6.20 & 0.75 \\
6.5 & 0 & 0.45 & 6.01 & 0.74 \\
7.0 & 0 & 0.24 & 0.00 & 0.23 \\
4.5 & 40 & 0.29 & 0.00 & 0.33 \\
5.0 & 40 & 0.22 & 0.00 & 0.15 \\
5.5 & 40 & 0.21 & 0.00 & 0.15 \\
6.0 & 40 & 0.21 & 0.00 & 0.15 \\
6.5 & 40 & 0.21 & 0.00 & 0.14 \\
7.0 & 40 & 0.37 & 2.83 & 0.52 \\
4.5 & 20 & 0.30 & 0.79 & 0.35 \\
4.5 & 30 & 0.32 & 1.12 & 0.39 \\
7.0 & 20 & 0.27 & 0.51 & 0.29 \\
7.0 & 30 & & & \\
\hline
\end{tabular}

Table 1. Volume-weighted mean diameter $\left(D_{4,3}, \mu \mathrm{m}\right)$, volume percentage of particles exceeding $1 \mu \mathrm{m}$ in diameter $\left(\% V_{d>1}\right)$, and width of the distribution $(W)$ of emulsions formulated with fish oil (10 wt.\%) as lipid phase and different concentrations of trehalose or sodium caseinate ( $\mathrm{NaCas}$ ) immediately after preparation.

In all cases, fish oil emulsions showed a monomodal distribution regardless of sodium caseinate concentration. However, $D_{4,3} \% V_{\mathrm{d}>1}$, and $W$ significantly decreased as sodium caseinate concentration increased indicating that protein concentration limited the fat globule size in this concentration range. Emulsions with trehalose also showed a monomodal distribution. $D_{4,3}$ was always smaller than for the emulsions without sugar in the aqueous phase showing that trehalose had strong interactions with the protein influencing droplet size. Besides, distribution for emulsions with trehalose (specially $40 \mathrm{wt} . \%$ ) were very narrow.

The Turbiscan equipment has a reading head which is composed of a pulsed near-IR light source $(\lambda=850 \mu \mathrm{m})$ and two synchronous detectors. The transmission detector receives the light, which goes through the sample $\left(0^{\circ}\right)$, while the back-scattering detector receives the light back-scattered by the sample $\left(135^{\circ}\right)$. The curves obtained by subtracting the BS profile at $\mathrm{t}=0$ from the profile at $\mathrm{t}$, that is $\triangle \mathrm{BS}=\mathrm{BS}_{\mathrm{t}}-\mathrm{BS}_{0}$, display a typical shape that allows a better quantification of creaming, flocculation and other destabilization processes. Creaming was detected using the Turbiscan as it induced a variation of the concentration between the top and the bottom of the cell. The droplets moved upward because they had a lower density than the surrounding liquid. When creaming take place in an emulsion, the $\triangle \mathrm{BS}$ curves show a peak at heights between $0-20 \mathrm{~mm}$. Flocculation was followed by measuring the $\mathrm{BS}_{\mathrm{av}}$ as a function of storage time in the middle zone of the tube. The optimum zone was the one no affected by creaming (bottom and top of the tube), that is, the $20-50 \mathrm{~mm}$ zone. Figure 5 reports as an example, changes in back scattering (BS) profiles (expressed in reference mode, $\triangle B S$ ) as a 
function of the tube length with storage time (samples were stored for 1 week, arrow denotes time) in quiescent conditions for emulsions with fish oil as fat phase, no trehalose added to the aqueous phase, and different concentrations of sodium caseinate: A) $4.5 \mathrm{wt} . \%, \mathrm{~B}) 5.0 \mathrm{wt} . \%, \mathrm{C})$ $5.5 \mathrm{wt} . \%$, D) $6.0 \mathrm{wt} . \%$, E) $6.5 \mathrm{wt} . \%$, and F) $7.0 \mathrm{wt} . \%$. All emulsions but the one stabilized with $6.0 \mathrm{wt} . \%$ of sodium caseinate destabilized by flocculation as indicated by the decrease in BS in the central part of the tube $(20-50 \mathrm{~mm})$. The $6.0 \mathrm{wt}$.\% emulsion was more stable. However, a slightly creaming was detected by Turbiscan as evidenced by the decreased of the profile at the bottom of the tube (zone $0-20 \mathrm{~mm}$ ) and the increase at the top of the tube. The stability zone is very narrow for emulsions without trehalose.

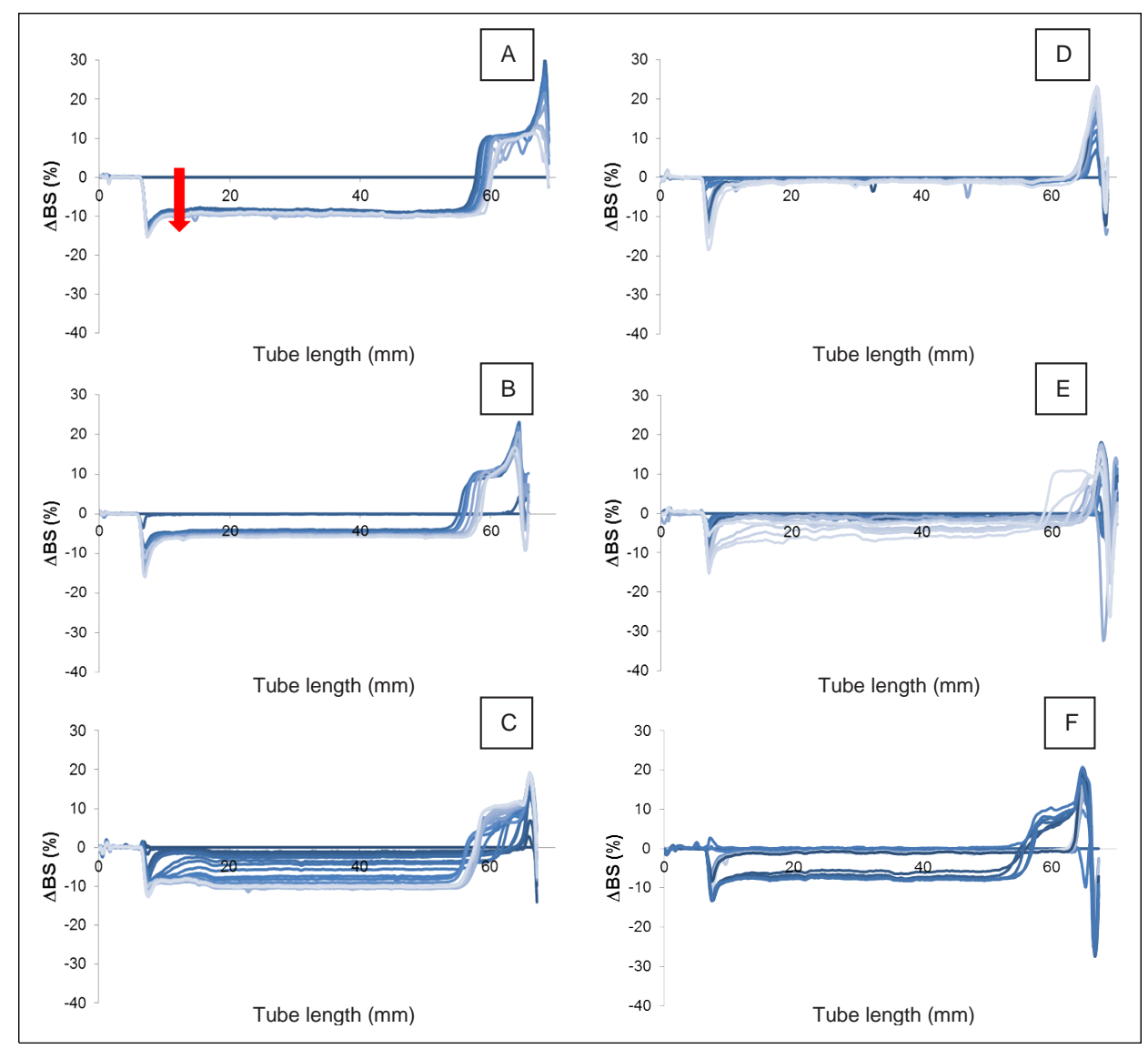

Figure 5. Changes in back scattering profiles in reference mode, as a function of the tube length with storage time (the emulsion was stored for 1 week, arrow denotes time) in quiescent conditions. It was formulated with 10 wt. \% fish oil as fat phase, no trehalose added to the aqueous phase, and a concentration of sodium caseinate of A) $4.5, B$ ) 5.0, C) 5.5, D) 6.0 , E) 6.5 , and F) 7.0 wt.\%. Tube length $65 \mathrm{~mm}$. 

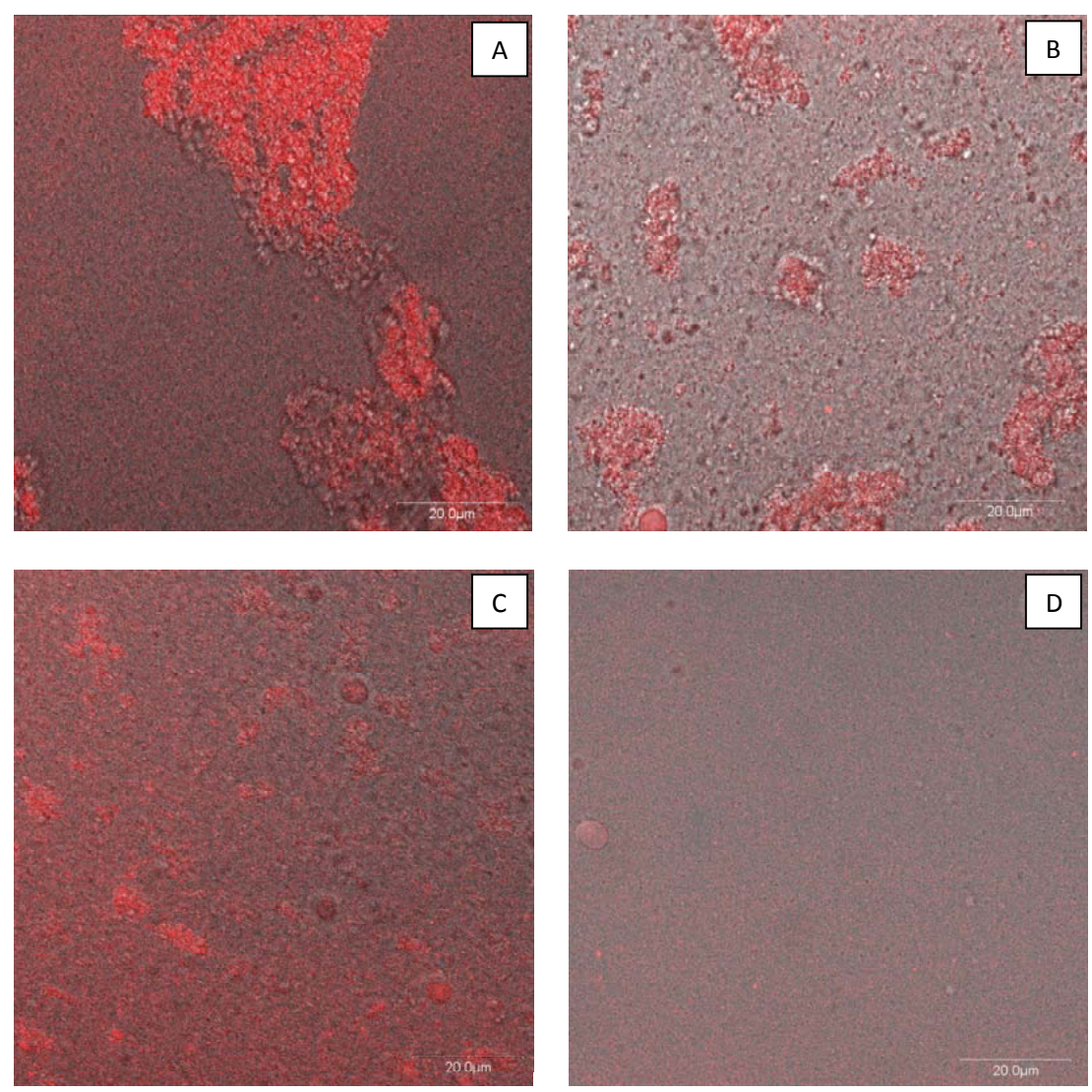

Figure 6. CLSM images of emulsions formulated with 10 wt.\% fish oil, 4.5 wt.\% sodium caseinate and different concentrations of trehalose: A) 0, B) 20, C) 30, and D) 40 wt.\%.

When 40 wt.\% trehalose was added to the aqueous phase of emulsions in Figure 5 the rate of destabilization was markedly lower. The Turbiscan analysis showed profiles similar than the one reported in Figure 5 D. The emulsions stabilized with $4.5 \mathrm{wt} . \%$ sodium caseinate or more did not flocculate during a week at $22.5^{\circ} \mathrm{C}$. $\triangle \mathrm{BS}$ profiles in the central zone of the tube (20-50 $\mathrm{mm}$ ) did not change during that time. These results were in agreement with the decrease in $D_{4,3}$ showed in Table 1 . It was reported that polysaccharides which are not particularly surface active such as xanthan gum and which are usually added to the aqueous phase of emulsions as thickening agents to retard instability mechanisms did not affect the size of the emulsion droplets. On the contrary, the fact that particle size diminished for sugar addition does not allow disregarding interactions. In some systems droplet size can be smaller if polysaccharides are present with the protein during homogenization, so the rate of creaming can be reduced as long as there is no bridging flocculation. Interactions between polysaccharides and proteins are based on hydrogen bond, and dipole-dipole associations, in which the presence of $\mathrm{OH}$ - 
groups plays a predominant role. Beside, in the Biochemistry field, strong interactions between peptides and short oligosaccharides have been recently reported as playing an important role in protein recognition. All the above indicates that sugar-protein interactions are very common. Regarding trehalose special properties described in literature it is not unreasonable to think that trehalose may work as coadjutant in micelle formation and, also, may change the quality of water as a solvent, improving the solvent-protein interaction, leading to smaller particles sizes and more stable emulsions.

To further explore this hypothesis, emulsions structure were studied by CLSM. Figure 6 reports CLSM images of emulsions formulated with $10 \mathrm{wt} . \%$ fish oil, $4.5 \mathrm{wt}$.\% sodium caseinate and different concentrations of trehalose: A) 0, B) 20, C) 30, and D) $40 \mathrm{wt} . \%$. As may be noticed from the CLSM images of Figure $6 \mathrm{~A}$, when the aqueous phase did not contain trehalose the resulting emulsion destabilized by flocculation. The Turbiscan profile of this emulsion was similar than the one showed in Figure 5 A. In agreement with the Turbiscan profile, CLSM detected flocs formation in this sample. When sugar was added microstructure changed being the changes more evident as trehalose concentration increased. Flocs diminished their size with sugar addition (Figure $6 \mathrm{~B}$ and C). When trehalose concentration was $40 \mathrm{wt} . \%$, single drops with a smaller size were noticeable in CLSM image (Figure 6 D). This more uniform microstructure was indicative of a greater stability. These changes in microstructure, qualitatively detected by CLSM, were quantified by measuring droplet size distribution by dynamic light scattering techniques (Table 1) and evaluating the Turbiscan profiles. These quantitative results were in agreement with the description provided by CLSM images, i.e., image reported in Figure 6 A corresponds to the Turbiscan in Figure 5 A. It is clear in Figure 6 A that emulsion structure is formed by flocs. This was the expected result from the profile in Figure $5 \mathrm{~A}$ that corresponds to an emulsion which main mechanism of destabilization is flocculation. The Turbiscan profile corresponding to the CLSM image in Figure $6 \mathrm{D}$ was similar to the one reported in Figure 5D. In agreement with Turbiscan measurements the microstructure of this emulsion (Figure 6 D) shows a homogeneous structure.

Figure 7 reports CLSM images of emulsions formulated with $10 \mathrm{wt}$ \% fish oil, $7 \mathrm{wt}$ \% sodium caseinate and different concentrations of trehalose: A) 0, B) 20, C) 30, and D) $40 \mathrm{wt} . \%$. All these microstructures corresponded to homogeneous emulsions with small droplets evenly distributed. As expected from CLSM images, the quantification of all systems by Turbiscan showed that they were very stable. Besides, in agreement with their microstructures, these emulsions showed a monomodal and very narrow distribution with a small $D_{4,3}$ when analyzed by dynamic light scattering (Table 1).

In order to slow down the destabilization of emulsions, thickening agents such as polysaccharides and hydrocolloids are frequently used. In Álvarez Cerimedo et al. study [17] it was shown that the effect of trehalose was further than the ability to form viscous solutions since it diminished average particle size values for the same processing conditions. The interactions between protein and sugar also played an important role in stabilization although was not enough to suppress the depletion effect that led to instability of the emulsions formulated with sodium caseinate concentrations from 2 to $4 \mathrm{wt}$.\%. Although sucrose has a different structure than trehalose, with the monosaccharides units bound C1-C4, according to our results, it seems 

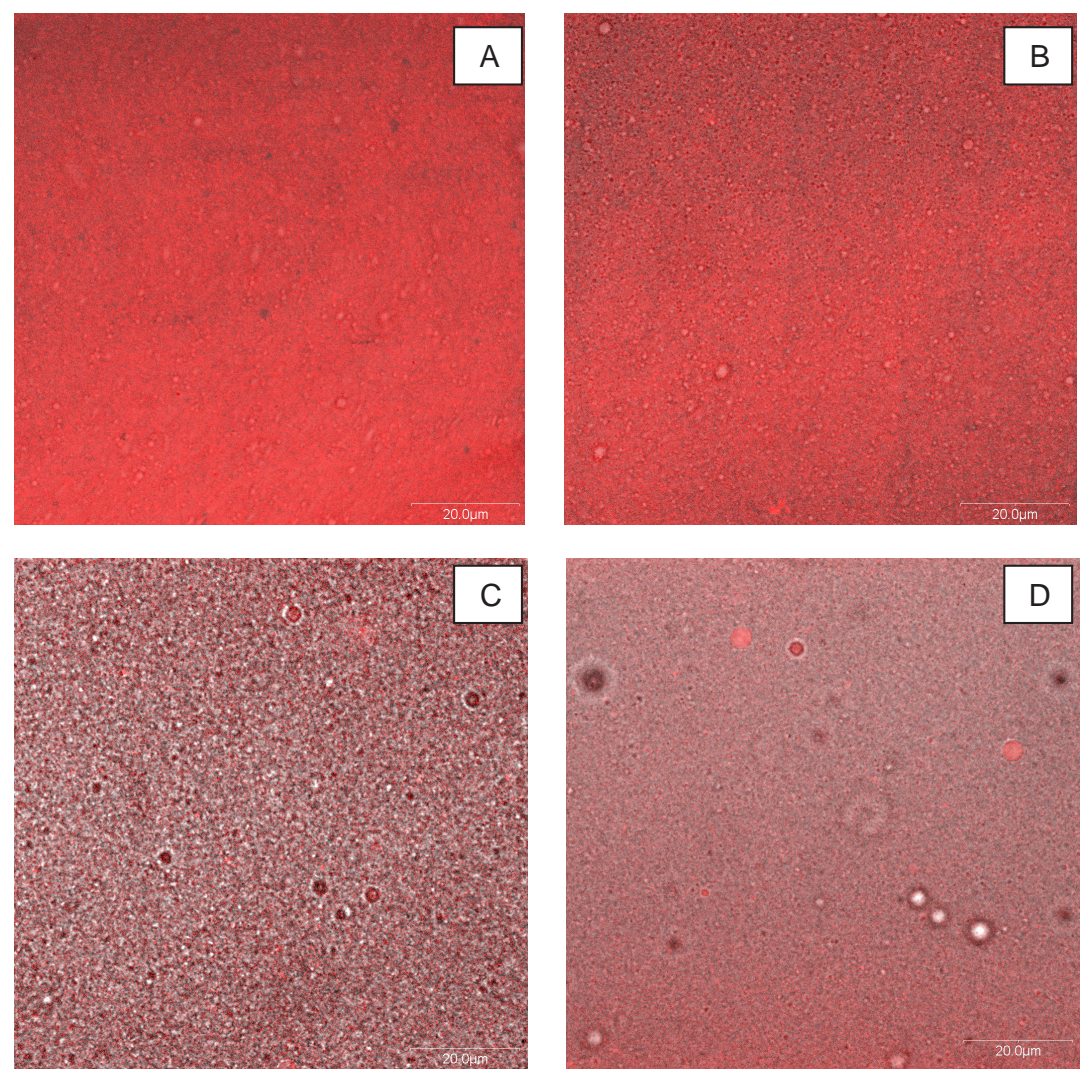

Figure 7. CLSM images of emulsions formulated with 10 wt. \% fish oil, 7 wt. \% sodium caseinate and different concentrations of trehalose: A) 0, B) 20, C) 30, and D) 40 wt.\%.

to have the same microstructural properties. It was reported that there was a pronounced dissociation of sodium caseinate sub-micelles in the presence of sucrose at a $\mathrm{pH}$ above the protein's isoelectric point due, most likely, to direct hydrogen bonding between sodium caseinate and sucrose. The dissociation of sodium caseinate sub-micelles was in excellent agreement with the more homogeneous microstructure and the formation of smaller compact protein structures as detected by CLSM.

\subsection{Combination of CLSM and other techniques}

Other applications of CLSM in emulsions in combination with other techniques are as follows:

Emulsions were also used to encapsulate lipids. Emulsion-based delivery systems have been developed to increase the oral bioavailability of lipophilic compounds within the gastrointestinal tract. They have also been used to control the release of lipophilic agents at specific locations within the gastrointestinal tract, such as the mouth, stomach, small intestine, or colon. 
Emulsion-based delivery systems typically utilize relatively small lipid droplets $(\mathrm{d}=10 \mathrm{~nm}-$ $100 \mu \mathrm{m}$ ) to contain the lipophilic components. The functionality of this type of delivery system can be tailored by controlling their composition and structural organization. This has led to the development of a number of different categories of emulsion-based delivery systems, including conventional emulsions, nanoemulsions, multilayer emulsions, solid lipid nanoparticles, multiple emulsions, microcluster emulsions, and filled hydrogel particles. Each of these systems has its own specific advantages and disadvantages for controlling lipid digestion and for releasing lipophilic components. Li et al. [18] evaluated the performance of four emulsion-based delivery systems with different structures: (A) conventional emulsions; (B) small microcluster emulsions; (C) large microcluster emulsions; (D) filled hydrogel beads. The fate of the delivery systems within the gastrointestinal tract was ascertained by introducing them into rat stomachs. Confocal microscopy showed that system D remained intact in the stomach, but systems A, B and C exhibited considerable disruption leading to droplet coalescence. This study showed that an in vitro digestion model is a useful predictive tool for in vivo feeding studies, and that encapsulation is an effective strategy to control the fate of lipids within the gastrointestinal tract. As mentioned, oil-in-water emulsions are widely used in the food industry to encapsulate lipophilic functional components, such as vitamins, colors, flavors, nutraceuticals, and antimicrobials. They are also used to provide desirable optical, rheological and sensory characteristics to many types of food products, including beverages, sauces, dips, dressings and deserts. Ziani et al. [19] investigated on the interaction of lipid droplets of emulsions with the surfaces of packaging materials, an area in which much less research was done. An improved understanding of the interactions of lipid droplets with packaging materials is important for a number of reasons. If lipid droplets are strongly attracted to the surface of a packaging material, then they may form a coating on the internal walls of the package. This coating may give an undesirable appearance to the product, particularly if the lipid droplets contain colorants. In addition, the lipid droplet concentration within the product will be depleted, which could also change the overall product appearance. The deposition of lipid droplets onto the container walls may also mean that any bioactive components encapsulated within them (such as vitamins or nutraceuticals) are not ingested when the product is consumed. On the other hand, the ability of lipid droplets to become attached to packaging surfaces may be desirable in other applications. For example, one or more layers of lipid droplets containing active ingredients could be deposited onto the surfaces of a packaging material to modify its functional characteristics, such as charge, optical properties, and antimicrobial activity. Ziani et al. [19] examined specifically the interaction between lipid droplets coming from a corn oil/water emulsion and polyethylene surfaces. They prepared lipid droplets with various surface charges in the presence of surfactants with different electrical characteristics: non-ionic (Tween 80), cationic (lauric arginate), and/or anionic (sodium dodecyl sulfate). The ionic properties of polyethylene surfaces were modified by UV treatment. Stable emulsions containing small droplets $(\mathrm{d}<200 \mathrm{~nm})$ with nearly neutral (Tween 80), cationic (Tween 80: lauric arginate), and anionic (Tween 80: sodium dodecyl sulfate) charges were prepared by adding different levels of the ionic surfactants to Tween 80 stabilized emulsions. Scanning electronic microscopy, confocal fluorescence microscopy, and ATR-FTIR showed that the number of droplets attached to the polyethylene surfaces depended 
on the droplet charge and the polyethylene surface characteristics. The greatest degree of droplet adsorption was observed for the cationic droplets to the UV-ozone treated polyethylene surfaces, which was attributed to electrostatic attraction. These results were a contribution to the understanding of the behavior of encapsulated lipophilic components in food containers. From Ziani et al. results [19], it seems to be possible to create novel functional properties of packaging materials by depositing lipid droplets containing active agents onto their surfaces, such as antimicrobials or antioxidants.

\subsection{Applications of CLSM in emulsion-based products}

Almonds are considered to be a great source of proteins, dietary fiber, health-promoting unsaturated fatty acids, vitamin E, other vitamins, minerals; they are also low in saturated fats, and contain no cholesterol. As a result, almonds are used in several food products, such as almond-based beverages, pastes, butter, snacks and baking goods. Lipids in almonds are present as oil bodies in the nut. These oil bodies are surrounded by a membrane of proteins and phospholipids and are a delivery vehicle of energy in the form of triglycerides, similarly to the more studied bovine milk fat globule membrane. Gallier et al. [20] performed chemical, physical and microscopic analyses of these systems. Their results revealed major differences in the composition and structure of almond oil bodies and bovine milk fat globules. The lipids of both natural emulsions differed in degree of unsaturation, chain length, and class. The almond oil body membrane does not contain any cholesterol or sphingomyelin unlike the bovine milk fat globule membrane. The membranes, a monolayer around almond oil bodies and a trilayer around bovine fat globules, may affect the stability of the lipid droplets in a food matrix and the way the lipids are digested.

The conversion of an emulsion into a stable foam requires the formation of a structure capable of retaining bubble integrity. It is well known that controlled destabilization of an emulsion by partial coalescence of fat droplets can provide the necessary means of foam stabilization. Dairy colloids such as whipped cream and ice cream are important examples of such aerated emulsions, being primarily stabilized by a matrix of partially aggregated fat globules at the air-water interface. Allen et al. [21] compared the aeration properties of acidified caseinstabilized emulsions containing liquid oil droplets to the whipping of dairy cream. The foam systems were characterized in terms of overrun, microstructure, drainage stability, and rheology. CLSM studies were performed staining protein and oil with Nile Blue and Nile Red, respectively. These dyes were incorporated into the emulsion immediately prior to whipping. Nile Red was used to stain the oil in whipped cream. Allen et al. [21] have found that a stable foam of high overrun can be made by aerating a sodium caseinate-stabilized emulsion which is undergoing gradual acidification. Such a foam, similar in fat content to whipped cream, yet containing only liquid oil droplets, is stabilized not by partial coalescence, but by aggregation of the protein-coat surrounding the droplets. Heuer et al. [22] performed further investigations on foam microstructure when subject to pressure change. They studied the static and dynamic effects of pressure on the stability of air bubbles to coalescence in process and formulation regimes that are relevant to much of the food industry. Analysis of the digital images obtained was then used to quantify the effects of pressure on bubble size distribution. According to 
these authors, the foam under study was relatively stable under quiescent conditions but could destabilize when subject to a pressure change similar to that of a typical industrial process.

Emulsions can be stabilized not only by surfactants, but also by solid particles, whence they are termed Pickering emulsions. Depending on their surface hydrophobicity, particles can stabilize Oil-in-Water, Water-in-Oil, or multiple emulsions. It is seen that the particles must be reasonably hydrophobic in order to adsorb but this also means that they will also have a tendency to aggregate in the aqueous phase. This aggregation may slow down the rate of droplet coverage by solid particles and curtail stable emulsion formation. However, for Oilin-Water emulsions there is the option of reducing the aggregation of the particles by dispersing them in the hydrophobic (oil) phase before emulsification. Yusoff and Murray [23] created hydrophobic starch particulates from starch granules (i.e., not free hydrophobic starch molecules) and tested their ability to stabilize model Oil-in-Water emulsions, optimizing the modification required for the formation of new and improved surface active agents. The physical properties (particle size and surface activity) and emulsifying properties (emulsion microstructure and stability) of the starch particulates were investigated. Conventional light transmission microscopy, CLSM, scanning electron microscopy, multi-angle light scattering and laser Doppler light scattering all suggested that a wide range of starch particle sizes was produced. Some particles were considerably smaller than the original starch granule sizes, but a large proportion appeared to be above several microns in size. The prepared emulsions creamed readily, but they were extremely stable to coalescence with no significant change in the emulsion droplet-size distributions for over 3 months.

Controlling the rate of droplet growth is critical for emulsion based products and processes. Particles added to Oil-in-Water emulsion formulations may attach to drops and impart kinetic stability for significant periods (weeks or longer). There is growing interest in how attached particle layers alter the rates of interfacial reactions and release of materials from drops by dissolution or evaporation. Little is known, however, about how particle-stabilized (Pickering) emulsions break. Predicting the lifetime of an emulsion and the processes by which it destabilizes remains challenging, even for surfactant-stabilized emulsions. Avendaño Juárez and Whitby [24] studied droplet evolution in unstable, dilute Oil-in-Water Pickering emulsions with the aim of knowing the processes of destabilization of an emulsion at low particle concentrations that do not kinetically stabilize emulsions. The systems under studied were toluene-in-water emulsions formed at low concentrations of silanised fumed silica nanoparticles. The emulsions were characterized using a combination of light scattering, CLSM and rheology. Their results showed that the particles coagulate in the aqueous phase of the emulsions, undergoing reaction limited, or slow, aggregation. The main cause of destabilization arises from aggregation of the particles into compact clusters that do not protect newly formed drops against flocculation. Transfer of oil between flocculated drops occurs by permeation of toluene molecules through the pores of the close packed particulate shells coating the drops. In contrast, particles form loose networks that keep the drops well separated in emulsions formed at higher particle concentrations. These findings show that the minimum particle concentration required to kinetically stabilize a Pickering emulsion is the concentration required for the particle aggregates to be connected into a single expanded network structure. 
Among proteins of vegetable origin, soy protein is an abundant byproduct of soybean oil industry and has good functional properties for food processing because of high nutritional value and the contribution to food texture and emulsifying properties. The isoelectric point of soy protein is about $\mathrm{pH} 4.8$, therefore, soy protein emulsions are unstable at $\mathrm{pH}$ around 5. As most foods and beverages are acidic, the poor emulsion stability at isoelectric point limits the applications of soy protein emulsions in food and beverage industries. The stability of protein emulsion can be improved by protein-polysaccharide conjugate produced via covalent bond or protein/polysaccharide complex formed by electrostatic attraction. Yin et al. [25] used a simple, green and effective strategy to produce long-term stable oil in water emulsion from soy protein and soy polysaccharide. Soy protein and soy polysaccharide formed dispersible complexes at $\mathrm{pH}$ around 3.25. A high pressure homogenization produced the protein/ polysaccharide complex emulsion having a droplet size about $250 \mathrm{~nm}$. A heat treatment of the emulsion resulted in the protein denaturation, forming irreversible oil-water interfacial films composed of soy protein/soy polysaccharide complexes. The droplets of the emulsion were characterized by dynamic light scattering, $\zeta$-potential, transmission electron microscopy, polysaccharide digestion via pectinase, and CLSM observation via dual fluorescence probes. As a result of the polysaccharide being fixed on the droplet surface, the emulsions exhibited long-term stability in the media containing $\mathrm{pH}$ values of $2-8$ and $0.2 \mathrm{~mol} / \mathrm{L} \mathrm{NaCl}$. According to Yin et al. [25], the stable soy protein/soy polysaccharide complex emulsion is a suitable foodgrade delivery system in which lipophilic bioactive compounds can be encapsulated.

The bioavailability of lipid components depends on their chemical structure, physicochemical properties, and the nature of the food matrix that surrounds them. In some situations it may be advantageous to increase the bioavailability of an ingested lipid, e.g., highly non-polar and crystalline bioactive components, such as carotenoids or phytosterols. In other situations, it may be more beneficial to decrease the bioavailability of an ingested lipid, e.g., saturated fats or cholesterol that can have a negative impact on human health. An improved understanding of the factors that impact the bioavailability of dietary lipids would enable the food industry to design foods to increase, decrease or control lipid digestion and absorption within the human gastrointestinal tract. Hur et al. [26] examined the impact of emulsifier type on the micro-structural changes that occur to emulsified lipids as they pass through a model gastrointestinal system. Lipid droplets initially coated by different kinds of emulsifiers (lecithin, Tween 20, whey protein isolate and sodium caseinate) were prepared using a high speed blender. The emulsified lipids were then passed through an in vitro digestion model that simulated the composition ( $\mathrm{pH}$, minerals, surface active components, and enzymes) of mouth, stomach and small intestine juices. The change in structure and properties of the lipid droplets were monitored by CLSM, conventional optical microscopy, light scattering, and microelectrophoresis. Nile red (a fat soluble fluorescent dye) was excited with $488 \mathrm{~nm}$ argon laser line. The general shape of the particle size distributions of all the emulsions remained fairly similar from initial-to-mouth-to-stomach, exhibiting a major population of large droplets and a minor population of smaller droplets. The largest change in mean droplet size occurred when the emulsions moved from the simulated stomach to small intestine, which might be attributed to digestion of the emulsified lipids by lipase and the incorporation of the lipid digestion 
products within mixed micelles and vesicles. In general, there was a decrease in mean droplet diameter $\left(d_{32}\right)$ as the droplets moved from mouth to stomach to small intestine.

\section{Gel-like emulsions}

Whey protein is a milk protein widely used in the food industry for gelation and texture formation. The aggregation and network formation of this globular protein have been previously well investigated in the bulk phase. Manoi and Rizvi [27] studied the emulsifying activity and emulsion stability indices of texturized whey protein concentrate and its ability to prevent coalescence of Oil-in-Water emulsions and compared its functionality with the commercial whey protein concentrate 80 . The cold, gel-like emulsions were prepared at different oil fractions $(\phi=0.20-0.80)$ by mixing oil with the $20 \%(\mathrm{w} / \mathrm{w})$ texturized whey protein concentrate dispersion at $25{ }^{\circ} \mathrm{C}$ and evaluated using a range of rheological techniques. Microscopic structure of cold, gel-like emulsions was also observed by CLSM. The results revealed that the texturized whey protein concentrate showed excellent emulsifying properties compared to the commercial whey protein concentrate 80 in slowing down emulsion breaking mechanisms such as creaming and coalescence. Very stable with finely dispersed fat droplets, and homogeneous Oil-in-Water gel-like emulsions could be produced. The emulsion products showed a higher thermal stability upon heating to $85^{\circ} \mathrm{C}$ and could be used as an alternative to concentrated Oil-in-Water emulsions and in food formulations containing heat-sensitive ingredients. Liu and Tang [28] also studied cold gel-like whey protein concentrate emulsions at various oil fractions ( $\phi: 0.2-0.6)$. Emulsions were formed through thermal pretreatment (at $70^{\circ} \mathrm{C}$ for $30 \mathrm{~min}$ ) and subsequent microfluidization. The rheogical properties and microstructures, as well as emulsification mechanism of these emulsions were characterized. CLSM analyses confirmed close relationships between rheological properties and gel network structures at various $\phi$ values. Chen and Dickinson [29] investigated surface properties of heatset whey protein gels $(14 \mathrm{wt} \%)$ using surface friction measurement and CLSM. The aims of that work was to establish reliable techniques for surface characterization of aggregated whey protein particle gels and to understand how the gel surfaces are affected by various influencing factors. In their studies, a He-Ne laser was used as the light source and fluorescence from the sample was excited at $543 \mathrm{~nm}$. Rhodamine B was used to stain protein and it was added after gelation. CLSM observations revealed that the protein gel without salt addition has a smooth and flat surface, while the gel with added salt has a much rougher surface.

Mixed biopolymer gels are often used to model semi-solid food products. Understanding of their functional properties requires knowledge about structural elements composing these systems. Thus, it is of prime importance to establish an in-depth understanding of the detailed nature and spatial distribution of structural elements included in the gels. Such information may also allow understanding the formation of protein/polysaccharide mixed gels and developing food products with tailored well-defined textural properties. Plucknett et al. [30] used CLSM to follow the dynamic structural evolution of several phase-separated mixed biopolymer gel composites. Two protein/polysaccharide mixed gel systems were examined: gelatin/maltodextrin and gelatin/agarose. These materials exhibit emulsion-like structures, 
with included spherical particles of one phase (i.e. polymer A) within a continuous matrix of the second (i.e. polymer B). Compositional control of these materials allows the phase order to be inverted (i.e. polymer B included and polymer A continuous), giving four basic variants for the present composites. For the micrographs presented in the current paper, the gelatinrich phase appears light, whereas both the maltodextrin-rich and agarose-rich phases appear dark. Tension and compression mechanical tests were conducted dynamically on the CLSM. This technique allowed describing microstructure under deformation. As a conclusion of the study it is postulated that polymer interdiffusion occurred across the interface for the gelatin/ agarose system, to a significantly greater extent than for interfaces between gelatin and maltodextrin, resulting in higher interfacial fracture energy. van den Berg et al. [31] also investigated mixed biopolymer gels. They described the structural features of mixed cold-set gels consisting of whey protein isolate and different polysaccharides (gellan gum, high methyl pectin or locust bean gum) at different length scales by using CLSM and scanning electron microscopy. Whey protein cold-set gels were prepared at different concentrations to emulate stiffness of various semi-solid foods. The gels were stained with an aqueous solution of Rhodamine B prior to gelation and allowed to gel inside a special CLSM cuvette. The dye binds non-covalently to the protein network. van den Berg et al. [31] described the structure of their systems with CLSM, at the micrometer length scale, showing that the gels had an homogeneous nature with a maximum resolution of around $1 \mu \mathrm{m}$. The CLSM images indicated that density of the protein network was proportionally related to the protein concentration. Going from low concentration to high concentration the pores within the protein network became smaller and disappeared at a protein concentration of $9 \%(\mathrm{w} / \mathrm{w})$. With decreasing pore size the density of the protein network increases. However, the morphology of whey protein isolates aggregates (i.e. size, shape and their connectivity) could not be described as it was below the resolution of CLSM. During the gel preparation (acidification), the presence of polysaccharides in the whey protein gels led to an initial phase separation into a gelled protein phase and a polysaccharide/serum phase at a micrometer length scale. At the final $\mathrm{pH}$ of the gels $(\mathrm{pH} 4.8$, i.e. below the $\mathrm{pI}$ of whey proteins), the negatively charged polysaccharides interacted with the protein phase and their spatial distribution was effected by charge density. Polysaccharides with a higher charge density were more homogeneously distributed within the protein phase. Neutral polysaccharide, locust bean gum, did not interact with the protein aggregates but was present in the serum phase. Whey protein gels were also analyzed by scanning electron microscopy. According to van den Berg et al. [31], a combination of the results of two microscopic techniques, CLSM and scanning electron microscopy, appeared to offer unique possibilities to characterize the structural elements of whey protein/polysaccharide cold-set gels over a wide range of length scales. Gaygadzhiev et al. [32, 33] also studied mixed biopolymer gels with focus on the impact of the concentration of casein micelles and whey proteinstabilized fat globules on the rennet-induced gelation of milk. The effect of different volume fractions of casein micelles and fat globules was investigated by observing changes in turbidity, apparent radius, elastic modulus and mean square displacement, in addition to CLSM imaging of the gels. Increasing the volume fraction of fat globules showed a significant increase in gel elasticity, caused by flocculation of the oil droplets. The presence of flocculated oil globules within the gel structure was confirmed by CLSM observations. Moreover, a lower degree of 
$\kappa$-casein hydrolysis was needed to initiate casein micelles aggregation in milk containing whey protein-stabilized oil droplets compared to skim milk. Gaaloul et al. [34] characterized the behavior of mixtures containing $\kappa$-carrageenan ( $\kappa$-car) and whey protein isolate using image analysis method. Heat treatment of whey protein isolate/k-car mixtures induces protein gelation and a phase-separation process. This is due to a modification in protein conformation. CLSM observations revealed the appearance of protein aggregate domains when phase separation occurred, with microgel droplets of whey protein isolate included in a continuous $\kappa$-car phase.

\section{Applications of CLSM to different products}

\subsection{Low fat products}

Industry is following the present trend of the market in developing low-fat products without changing the sensory and techno-functional properties of semi-solid milk products. Initially, hydrocolloids and stabilizers were used to imitate the fat perception and to enhance the stability of yoghurt regarding syneresis during storage. A reduced fat content caused a loss in viscosity and structure resulting in an altered appearance, texture, and mouthfeel. An alternative to hydrocolloids and stabilizers is the use of milk ingredients like whey proteins arising as a coproduct in the dairy industry. Krzeminski et al. [35] studied the effect of whey protein addition on structural properties of stirred yoghurt systems at different protein and fat content using laser diffraction spectroscopy, rheology and CLSM. CLSM images illustrated that the presence of large whey protein aggregates and lower number of fat globules lead to the formation of an interrupted and coarse gel microstructure characterized by large interstitial spaces. The higher the casein fraction and/or the fat level, the less interspaced voids in the network were observed. However, according to Krzeminski et al. [35], it is evident that the addition of whey proteins reinforces firmness properties of low-fat yoghurts comparable to characteristics of full-fat yoghurt. Celigueta Torres et al. [36] also studied differences in the microstructure of low fat yoghurt manufactured with microparticulated whey proteins used as fat replacer. One commercial and three experimental microparticulated ingredients with different chemical characteristics were used in the yoghurt formulations and compared to both full and low fat yoghurts without fat replacer. The results showed that the amount of native and soluble whey proteins present in the microparticles had a positive influence on the structure of the formed gel. The created structure, dominated by dense aggregates and low amount of serum, had an increased degree of self similarity or fractality with yoghurts in which fat was present. Gel-like emulsions are very interesting systems since they may be applied in food formulations as a kind of carrier for heat-labile and active ingredients. Studying similar systems, Le et al. [37] described the physical properties and microstructure of yoghurt enriched with milk fat globule membrane material. In milk, lipids are present in the form of globules with a diameter varying from 0.1 to $10 \mathrm{~mm}$. These fat globules have a surrounding thin membrane called the milk fat globule membrane. Fat in the core of the globules is mainly composed of neutral triacylglycerides whereas the lipid fraction in the milk fat globule membrane mainly comprises polar lipids. In view of its nutritional and technological proper- 
ties, milk fat globule membrane material has a high potential to act as a novel ingredient for the development of new functional food products. From an economic point of view, byproducts in dairy processing still have a lower price compared with main-stream products. The utilization of these sources to isolate the functional milk fat globule membrane material, and then apply it in the production of new products, may bring great benefit. Le et al. [37] studied applications of milk fat globule membrane in food product development such as enriched yoghurt. Milk fat globule membrane material was isolated from industrial buttermilk powder and both were used as a supplement for the production of yoghurt. Milk fat globule membrane isolated from BMP contained a high concentration of skim milk proteins. Based on confocal laser scanning microscopy, the microstructure of milk fat globule membrane-enriched yoghurts was denser from that of plain skim milk and buttermilk powder-enriched yoghurts. These results indicate the high potential of milk fat globule membrane material to be used as a novel ingredient for the development of new functional products, utilizing both the technological functionalities as well as the nutritional properties of the material.

\subsection{Starch-based products}

Starch represents the major storage product of most plants. In contrast to the transient starch found in photosynthetic tissues, storage starch accumulates in the plastids of starch storing tissues such as tubers and seeds over long periods of time to form large, well-organized granular structures. Starch granules are made up of two structurally distinct polymers of glucose. Amylose constitutes about $20-40 \%$ of typical storage starch and amylopectin constitutes the remaining $60-80 \%$ of the granule and is a much larger and highly branched polymer. The branch points are not randomly distributed but are clustered into an ordered arrangement allowing adjacent linear segments to form double helices. It is now widely accepted that the double helices formed by interacting chains of amylopectin form the basis for the crystalline structure of starch and are ordered into concentric crystalline lamellae interrupted by amorphous lamellae containing the branch points. Glaring et al. [38] used CLSM imaging and 8amino-1,3,6-pyrenetrisulfonic acid (APTS) as a probe for starch molecule distribution and ProQ Diamond as a specific probe for phosphate. In conjunction with CLSM they described their systems with scanning electron microscope. The aim of their work was to study the relationship between internal and external structural features of starches extracted from different botanical sources and genetic backgrounds. The investigation has focused on characterizing the genotype-specific internal deposition of starch molecules and how this is manifested at the surface of the granule. By using a combined surface and internal imaging approach, interpretations of a number of previous structural observations is presented. In particular, internal images of high amylose maize and potato suggest that multiple initiations of new granules are responsible for the compound or elongated structures observed in these starches. CLSM optical sections of rice granules revealed an apparent altered distribution of amylose in relation to the proposed growth ring structure, hinting at a novel mechanism of starch molecule deposition. Well-described granule features, such as equatorial grooves, channels, cracks, and growth rings were documented and related to both the internal and external observations. Ishihara et al. [39] used CLSM to visualize the degree of starch gelatinization and the distribution of gum Arabic and soybean soluble polysaccharide in the starch/polysaccharide composite system 
using fluorescein isothiocianate and rhodamine B as fluorescent dye. The aim of their work was to improve the quality, overall palatability and commercial value of rice-based food products by using novel texture modifiers. CLSM allowed them to see both the starch granules and the glutinous layer around the surface of the starch granules as a result of gelatinization in the presence and the absence of gum Arabic and soybean soluble polysaccharide. According to their results, soybean soluble polysaccharide was more effective in lowering the degree of starch gelatinization than gum Arabic in terms of granular size and the volume of the glutinous layer. Their images showed that the distribution pattern was different between these polysaccharides. Gum Arabic was present in a dispersed and scattered manner while soybean soluble polysaccharide covered in a continuous way the surface of the starch granules. Trivedi et al. $[40,41]$ processed a range of commercial cheese samples containing starch on a Rapid Visco Analyser (RVA) and on a pilot plant scale. This work clearly demonstrated that it was possible to manufacture processed cheese with part of the protein replaced with potato starch, while maintaining similar rheological attributes (firmness) to those of the control and an acceptable melt index. The confocal micrographs of the processed cheeses prepared in the pilot plant suggested that the fat particle size decreased as the starch levels increased. The reduction in fat particle size would then contribute to increase firmness and decrease melt. Sensory evaluation showed that, although the reduced-protein cheese samples had a good, clean, fresh flavour that was comparable with that of the control, at high starch concentrations the starchcontaining processed cheese had a pasty texture and tended to stick to the wrapper.

\subsection{Processed potatoes}

Potatoes (Solanum tuberosum L.) are an important source of carbohydrates and consumed widely in developing, as well as the developed world. Morphologically, a potato tuber is usually oval to round in shape with white flesh and a pale brown skin, although variations in size, shape, and flesh color are frequently encountered. The color, size, and cooked potato texture are the main quality attributes assessed by consumer for the acceptability of potatoes at domestic scale. However, a quality screening for the industrial processing of potatoes include several parameters, such as dry matter, starch content and characteristics, post harvest, and post processing shelf stability. Texture is one of the most essential technological quality attributes in processed potatoes. It is affected by raw material properties and processing conditions including salting of potatoes and cooking conditions. As salting of potatoes is an important issue for industrially processed potatoes, Straadt et al. [42] investigated the influence of salting on changes in texture, microstructure and water mobility and distribution in two raw material qualities of potatoes presented by two dry matter fractions within one variety. This study was the first report of the combined use of low-field nuclear magnetic resonance and CLSM in the study of the effects of salting on low and high dry matter potato tissue. The simultaneous use of CLSM and low-field nuclear magnetic resonance resulted in important information in relation to the interpretation of the origin of the low-field nuclear magnetic resonance water populations. Salting caused the raw potato cells to loose weight, which in the microscopic images was observed as loss of turgor pressure still further away from the edges of the samples with increased salting time. The paper illustrates the aptitude of low-field nuclear magnetic resonance and CLSM to determine and elucidate structural changes and 
associated changes in water mobility in potato tissue. Bordoloi et al. [43] described cooking, microstructural and textural characteristics from four New Zealand potato cultivars (Agria, Nadine, Moonlight, and Red Rascal). Potatoes from the waxy cultivar, Nadine, showed lowest dry matter and starch content and also had highest cooking time compared to the other cultivars. CSLM micrographs revealed Moonlight and Red Rascal raw potato parenchyma cellular structure to be well integrated, showing compact hexagonal cells. Raw tubers from these cultivars also exhibited higher hardness and cohesiveness, as observed using texture profile analysis. Moonlight potato parenchyma retained cell wall outline after cooking and its cells were observed to be completely filled with gelatinized starch matrix, whereas the cellular structure of Nadine potato parenchyma was completely disintegrated after cooking.

\subsection{Chocolate}

In addition to milk fat, another possible source of trapped fat in chocolate is from the cocoa ingredients, which may be in the form of cocoa mass, often also referred to as cocoa liquor, or cocoa powder. The total fat content of cocoa mass is typically about $55 \mathrm{~g} / 100 \mathrm{~g}$, while it is between 11 and $22 \mathrm{~g} / 100 \mathrm{~g}$ for standard cocoa powders and less than $1 \mathrm{~g} / 100 \mathrm{~g}$ for a highly defatted cocoa powder. The fat content of the cocoa ingredient counts towards the fat content of chocolate, which is usually around $30 \mathrm{~g} / 100 \mathrm{~g}$. A chocolate formulated with a cocoa solid of high trapped fat content contains less free fat which affects the viscosity behavior of the chocolate in its molten state since only the free fat 'contributes' to the chocolate's flowability. A certain level of flowability is required to facilitate processing such as pumping, conching, molding and enrobing as well as to impart an acceptable mouthfeel. Therefore, in an attempt to formulate chocolate with a lower fat content, simply decreasing the level of fat is not a valid approach. Aiming at the manufacture of reduced fat chocolates Do et al. [44] developed a novel method of trapped fat reduction: manipulation of the cocoa ingredient. Cocoa mass was replaced with cocoa powder (11 g/100 g or $<1 \mathrm{~g} / 100 \mathrm{~g}$ fat) and added 'free' cocoa butter. CLSM was used to visualize the internal microstructure of cocoa particles paying particular attention to fat partitioning. The approach was to let fluorescently stained oil diffuse into the particles to identify (i) the porous microstructure and (ii) the presence of fat inclusions on the CLSM micrographs acquired. Imaging of the cocoa solids was performed on the raw material as obtained. Results showed that the cocoa mass chocolate had a higher viscosity than cocoa powder chocolates of the same total fat content due to the presence of trapped fat globules as identified by CLSM. Based on the evidence presented, it can be concluded that standard defatted cocoa powder, as widely used by the industry, is indeed the best compromise in terms of free fat, particle size and morphology attempting to formulate fat reduced chocolate of acceptable molten state viscosity. Svanberg et al. [45,46] also studied changes in chocolate structure with formulation. They described the effect of major chocolate ingredients (sugar, cocoa particles and lecithin), in combination with the two pre-crystallization techniques, seeding and non-seeding, on the kinetics of cocoa butter crystallization and the resulting microstructure. CLSM was used to monitor microstructural evolution under dynamic thermal conditions. Differential scanning calorimetry measurements and image analysis were also applied in order to quantify the impacts of processing and formulation on microstructure. Segmentation between solid particles (i.e., sugar and cocoa particles, crystalline and liquid 
cocoa butter) was made through manual thresholding, which shows solid particles as black and liquid fat as significantly bright, allowing it to be distinguished from the crystals. Both the pre-crystallization techniques and the ingredients proved to have a significant impact on the kinetics and morphology of cocoa butter crystallization, and substantially different microstructures were observed. Complementary differential scanning calorimetry measurements were performed to estimate the amount of stable versus non-stable crystals present in the samples. These calculations also displayed large variations between seeded and non-seeded samples, as well as between the additions of ingredients, especially lecithin.

\subsection{Cheese}

Many of the properties of cheese such as cheese texture and flavour are determined by the spatial arrangement of components including: the casein particles that form a protein matrix, the fat globules, dispersed water and minerals. The arrangement of these components on the micron scale is known as the microstructure of cheese. In Cheddar cheese, this microstructure develops when milk coagulates to form a gel, usually through the enzymatic action of chymosin. Changes in process conditions or the choice of ingredients can alter the microstructure of the gel, curd and cheese considerably and thus the functional properties of the final cheese product. The ability to image and characterize these changes will provide an important tool for the quality control of cheese and other dairy products. Ong et al. [47] used cryo scanning electron microscopy and CLSM to visualize changes in the microstructure of milk, rennet-induced gel and curd during the manufacture of Cheddar cheese. When the structure of the gel, curd and cheese was observed using CLSM, spherical fat globules were mostly present in the serum pores of the gel prepared from unhomogenized milk but were found embedded in the aggregated chains of the casein network within the gel when prepared from homogenized milk. The porosity measurements obtained using cryo SEM were similar to those obtained using CLSM. These two complementary techniques can potentially be used to assist studies for the control of cheese texture and functionality. Costa et al. [48] investigated the effect of an exopolysaccharide-producing starter culture on milk coagulation and curd syneresis during the manufacture of half-fat Cheddar cheese by comparing the effect of an exopolysaccharide-producing starter with its non exopolysaccharide-producing isogenic variant. Coagulation and syneresis were monitored using light backscatter sensor technologies and the charge of the exopolysaccharide was also established. The distribution of the exopolysaccharide within the cheese microstructure was investigated by staining with Concavalin A and using CLSM. The study indicated that the exopolysaccharide produced by this starter culture did not interfere with coagulation but had a significant effect on reducing syneresis shortly after cutting. The exopolysaccharide was observed to be uniformly distributed throughout the cheese matrix and specifically located near the aqueous pores, possibly binding moisture and causing the observed decrease in syneresis.

Oiling-off is a major phenomenon that occurs during heating of semi-hard and hard-cheeses. The formation of a thin layer of free oil on the surface of the pieces of cheeses is required to prevent moisture evaporation during cooking and skin formation that impairs flow and stretch. Free oil lubricates the displacement of the para-casein matrix and has been described 
as the driving force of cheese melting. Oiling-off is also involved in the browning properties of cheeses which are heated for culinary applications. Indeed oiling-off may modulate dehydration and water activity, which is well known to strongly influence Maillard reactions. However, excessive oiling-off leads to appearance defects such as bi-phasing and to excessive chewiness of partially defatted melted cheese. Richoux et al. [49] investigated the influence of temperature and pressing strength on the oiling-off of Swiss cheese. Twin-cheeses were submitted to various cooling rates after the curd have been dipped from the vat. These cheeses were either pressed or non-pressed. Samples have been characterized using CLSM and were double stained: the fat phase was coded in red and the protein phase was coded in green. Aqueous phase appears as black areas in the CLSM images. Using this strategy, authors showed that a higher heat load yields to larger inclusions of fat in the cheese matrix and to higher oiling-off. The heat load applied to the curd (integral of the temperature vs. time curve) and oiling-off of cheeses aged of 5 days were linearly related. In contrast, the pressing strength did not influence oiling-off. As a conclusion, the heat load applied during pressing and acidification could be a tool to modulate the oiling-off of Swiss cheese.

\subsection{Fried food}

The market for fried foods remains very large and it continues to increase although there are health concerns associated with consuming high calorie foods. The peculiar organoleptic properties of fried foods such as good mouth-feel, distinct flavor, unique taste and palatability make them irresistible. Application of batter and breading coating is one of the means devised to reduce fat uptake during the frying operation aside from the fact that they add more value such as improved texture, appearance, taste and volume, to fried foods. The structure that is developed during processing to a large extent, defines some of the quality attributes of the coating system. Food structure can be characterized in terms of density, porosity, pore size distribution and specific volume. Porosity and pore size distribution are very important microstructural properties of fried foods needed in process optimization and product development. Adedeji et al. [50] characterized the pore properties and quantify fat distribution in deep-fat fried chicken nuggets batter coating using CLSM. Images were obtained at fluorescence mode with excitation/emission wavelengths of $488 / 570 \mathrm{~nm}$ to show fat distribution, and reflection/trans mode to obtain grayscale images which show structure of the sample that include pore distribution. Fat distribution obtained from image analysis was significantly (P $<0.05$ ) affected by the frying temperature and time, and it decreased within the depth of the sample thickness. Pore size varied approximately between 1.20 and $550 \mu \mathrm{m}$. Frying process led to the formation of more micropores (pores $<40 \mu \mathrm{m}$ ) and bigger (pore $\geq 216 \mu \mathrm{m}$ ) pores.

\subsection{High-protein snack bars}

Many processed foods are multicomponent heterogeneous systems that are far from thermodynamic equilibrium. There is often a considerable time lag between manufacture and consumption, during which a product is transported and stored. During this storage time, multiple chemical, physical, and biological reactions occur serially and simultaneously. Some of these reactions lead to the development of desirable attributes, such as good flavors in aged wines and cheeses, but others create flavors, colors, or textures that impact negatively on 
quality, as perceived by consumers. The shelf life can be defined as the length of time for which a product can be stored before the appearance of the first characteristic that consumers find unappealing, e.g., texture that is too tough. High-protein snack bars (hereafter called 'protein bars') are a convenient and nutritious food format that was originally developed for athletes but is now formulated to appeal to a wide range of health-conscious consumers. These bars contain $15-35 \%$ protein, which consists almost exclusively of dairy or soy proteins because of their health benefits and cost effectiveness. Chocolate, sugars, and flavorings create an appealing taste and flavor. Nuts, wafers, nuggets, etc. may be added for novel texture. Vitamins, minerals, and/or fiber are often added for enhanced nutritional value. Loveday et al. [51] examined the contributions of various chemical and physical reactions to the hardening of a model protein bar stored for up to 50 days at $20^{\circ} \mathrm{C}$. During manufacture of the protein bars, a subsample was withdrawn after the final mixing and a few drops of dye were added. The dye was a mixture of Nile Blue and Fast Green FCF dissolved at $0.2 \% \mathrm{w} / \mathrm{v}$ in a commercial antifading mountant medium, Citifluor (Citifluor Ltd., Leicester, UK). The dye was mixed into the protein bar material with a knife until even coloring was achieved, as judged by eye, and then a drop of the mixture was placed on a glass cavity slide and a coverslip was applied. The slides were stored at $20^{\circ} \mathrm{C}$. Over the first $17 \mathrm{~h}$ after manufacturing, protein particles became more clustered, and soluble protein appeared to precipitate, as shown by CLSM. Moisture migration induced important changes in molecular mobility and in microstructure.

\section{Conclusions}

Based on the above presented examples it can be concluded that CLSM broadens the application of conventional light microscopy. Combined with other techniques it is a valuable tool to describe a variety of food systems because it gives the possibility to examine the internal structureofthicksamplesinthreedimensions.Samplesmaybeanalyzed withoutperturbingthesystem and food transformations during processing may be followed in situ using specially designed stages. By using CLSMit is possible the simultaneous labeling of two or more components of food with probes which are specific for each component and therefore food structure may be described in more detail. A variety of model food systems was successfully analyzed, i.e., bulk fats, emulsions and gels. Besides, real products such asyoghurt, potatoes, chocolate, cheese, fried food and protein bar were also studied by CLSM in combination with other techniques given valuable data of food structure and its relationship with macroscopic properties.

\section{Acknowledgements}

This work was supported by CONICET through Project PIP 11220080101504, by the National Agency for the Promotion of Science and Technology (ANPCyT) through Project PICT 0060, and by the University of Buenos Aires through Project 20020100100467. 


\section{Author details}

Jaime A. Rincón Cardona ${ }^{1}$, Cristián Huck Iriart $^{2,3}$ and María Lidia Herrera ${ }^{3 *}$

*Address all correspondence to: lidia@di.fcen.uba.ar

1 School of Science and Technology, University of San Martín (UNSAM), San Martín City, Buenos Aires Province, Argentina

2 Institute of Inorganic Chemistry, Environmental Science and Energy (INQUIMAE), National Research Council of Argentina (CONICET), Buenos Aires City-State, Argentina

3 Faculty of Exacts and Natural Sciences (FCEN), University of Buenos Aires (UBA), Buenos Aires City-State, Argentina

\section{References}

[1] Marangoni A G, Hartel R W. Visualization and Structural Analysis of Fat Crystal Networks. Food Technology 1998;52(9) 46-51.

[2] Ding K, Gunasekaran S. Three-Dimensional Image Reconstruction Procedure for Food Microstructure Evaluation. Artificial Intelligence Review 1998;12(1-3) 245-262.

[3] Dürrenberger M B, Handschin S, Conde-Petit B, Escher F. Visualization of Food Structure by Confocal Laser Scanning Microscopy (CLSM). LWT - Food Science and Technology 2001;34(1) 11-17.

[4] Heertje I, van der Vlist P, Blonk J C G, Hendrickx H A C M, Brakenhoff G J. Confocal Scanning Laser Microscopy in Food Research: Some Observations. Food Microstructure 1987;6(2) 115-120.

[5] Blonk J C G and van Aalst H. Confocal Scanning Light Microscopy in Food Research. Food Research International 1993;26(4) 297-311.

[6] Herrera M L, Hartel R W. Unit D 3.2.1-6 Lipid Crystalline Characterization, Basic Protocole. In: Current Protocols in Food Analytical Chemistry (CPFA). New York: John Wiley \& sons, Inc.; 2001.

[7] Ong L, Dagastine R R, Kentish S E, Gras S L. Microstructure of Milk Gel and Cheese Curd Observed Using Cryo Scanning Electron Microscopy and Confocal Microscopy. LWT - Food Science and Technology 2011;44(5) 1291-1302.

[8] Auty M A E, Twomey M, Guinee T P, Mulvihill D M. Development and Application of Confocal Scanning Laser Microscopy Methods for Studying the Distribution of Fat and Protein in Selected Dairy Products. Journal of Dairy Research 2001;68(3) 417-427. 
[9] Herrera M L, Hartel R W. Effect of Processing Conditions on Physical Properties of a Milk Fat Model System: Microstructure. Journal of the American Oil Chemists' Society 2000;77(11) 1197-1204.

[10] Wiking L, De Graef V, Rasmussen M, Dewettinck K. Relations Between Crystallisation Mechanisms and Microstructure of Milk Fat. International Dairy Journal $2009 ; 19(8)$ 424-430.

[11] Martini S, Herrera M L, Hartel R W. Effect of Cooling Rate on Crystallization Behavior of Milk Fat Fraction/Sunflower Oil Blends. Journal of the American Oil Chemists' Society 2002;79(11) 1055-1062.

[12] Martini S, Puppo M C, Hartel R W, Herrera M L. Effect of Sucrose Esters and Sunflower Oil Addition on Microstructure of a High-Melting Milk Fat Fraction. Journal of Food Science 2002;67(9) 3412- 3418.

[13] Kaufmann N, Andersen U, Wiking L. The Effect of Cooling Rate and Rapeseed Oil Addition on the Melting Behaviour, Texture and Microstructure of Anhydrous Milk Fat. International Dairy Journal 2012;25(2) 73-79.

[14] Buldo P, Wikin L. The Role of Mixing Temperature on Microstructure and Rheological Properties of Butter Blends. Journal of the American Oil Chemists' Society 2012;89(6) 787-795.

[15] Hemar Y, Liu L H, Meunier N, Woonton B W. The Effect of High Hydrostatic Pressure on the Flow Behaviour of Skim Milk-Gelatin Mixtures. Innovative Food Science Emerging Technology 2010;11(3) 432-440.

[16] Radford S J, Dickinson E, Golding M. Stability and Rheology of Emulsions Containing Sodium Caseinate: Combined Effects of Ionic Calcium and Alcohol. Journal of Colloids and Interface Science 2004;274(2) 673-686.

[17] Álvarez Cerimedo M S, Huck Iriart C, Candal R J, Herrera M L. Stability of Emulsions Formulated with High Concentrations of Sodium Caseinate and Trehalose. Food Research International 2010;43(5) 1482-1493.

[18] Li Y, Kim J, Park Y, McClements D J. Modulation of Lipid Digestibility Using Structured Emulsion-Based Delivery Systems: Comparison of in Vivo and in Vitro Measurements. Food \& Function 2012;3(5) 528-536.

[19] Ziani K, Barish J A, McClements D J, Goddard J M. Manipulating Interactions Between Functional Colloidal Particles and Polyethylene Surfaces Using Interfacial Engineering. Journal of Colloid and Interface Science 2011;360(1) 31-38.

[20] Gallier S, Gordon K C, Singh H. Chemical and Structural Characterisation of Almond Oil Bodies and Bovine Milk Fat Globules. Food Chemistry 2012;132(4) 1996-2006. 
[21] Allen K E, Dickindon E, Murray B. Acidified Sodium Caseinate Emulsion Foams Containing Liquid Fat: A Comparison With Whipped Cream. LWT - Food Science and Technology 2006;39(3) 225-234.

[22] Heuer A, Coxa A R, Singleton S, Barigou M, van Ginkel M. Visualisation of Foam Microstructure When Subject to Pressure Change. Colloids and Surfaces A: Physicochemical and Engineering Aspects 2007;311(1-3) 112-123.

[23] Yusoff A, Murray B S. Modified Starch Granules as Particle-Stabilizers of Oil-in-Water Emulsions. Food Hydrocolloids 2011;25(1) 42-55.

[24] Avendaño Juárez J, Whitby C P. Oil-in-water Pickering Emulsion Destabilisation at Low Particle Concentrations. Journal of Colloids and Interface Science 2012;368(1) 319-325.

[25] Yin B, Deng W, Xu K, Huang L, Yao P. Stable Nano-Sized Emulsions Produced From Soy Protein and Soy Polysaccharide Complexes. Journal of Colloid and Interface Science 2012;380(1) 51-59.

[26] Hur S J, Decker E A, McClements D J. Influence of Initial Emulsifier Type on Microstructural Changes Occurring in Emulsified Lipids During in Vitro Digestion. Food Chemistry 2009;114(1) 253-262.

[27] Manoi K, Rizvi S S H. Emulsification Mechanisms and Characterizations of Cold, Gel-Like Emulsions Produced From Texturized Whey Protein Concentrate. Food Hydrocolloids 2009;23(7) 1837-1847.

[28] Liu F, Tang C H. Cold, Gel-Like Whey Protein Emulsions by Microfluidisation Emulsification: Rheological Properties and Microstructures. Food Chemistry 2011;127(4) 1641-1647.

[29] Chen J, Dickinson E. Surface Texture of Particle Gels. A Feature in Two Dimensions or Three Dimensions?. Chemical Engineering Research and Design 2005;83(7) 866870.

[30] Plucknett K P, Pomfret S J, Normand V, Ferdinando D, Veerman C, Frith W J, Norton I T. Dynamic Experimentation on the Confocal Laser Scanning Microscope: Application to Soft-Solid, Composite Food Materials. Journal of Microscopy 2001;201(2) 279-290.

[31] van den Berg L, Rosenberg Y, van Boekel M A J S, Rosenberg M, van de Velde F. Microstructural Features of Composite Whey Protein/Polysaccharide Gels Characterized at Different Length Scales. Food Hydrocolloids 2009;23(5) 1288-1298.

[32] Gaygadzhiev Z, Corredig M, Alexander M. The Impact of the Concentration of Casein Micelles and Whey Protein-Stabilized Fat Globules on the Rennet-Induced Gelation of Milk. Colloids and Surfaces B: Biointerfaces 2009a;68(2) 154-162. 
[33] Gaygadzhiev Z, Hill A, Corredig M. Influence of the Emulsion Droplet Type on the Rheological Characteristics and Microstructure of Rennet Gels From Reconstituted Milk. Journal of Dairy Research 2009b;76(3) 349-355.

[34] Gaaloul S, Turgeon S L, Corredig M. Phase Behavior of Whey Protein Aggregates/אCarrageenan Mixtures: Experiment and Theory. Food Biophysics 2010;5(2) 103-113.

[35] Krzeminski A, Großhable K, Hinrichs J. Structural Properties of Stirred Yoghurt as Influenced by Whey Proteins. LWT - Food Science and Technology 2011;44(10) 2134-2140.

[36] Celigueta Torres I, Amigo Rubio J M, Ipsen R. Using Fractal Image Analysis to Characterize Microstructure of Low-Fat Stirred Yoghurt Manufactured with Microparticulated Whey Protein. Journal of Food Engineering 2012;109(4) 721-729.

[37] Le T T, van Camp J, Pascual P A L, Meesen G, Thienpont N, Messens K, Dewettinck K. Physical Properties and Microstructure of Yoghurt Enriched with Milk Fat Globule Membrane Material. International Dairy Journal 2011;21(10) 798-805.

[38] Glaring M A, Koch C B, Blennow A. Genotype-Specific Spatial Distribution of Starch Molecules in the Starch Granule: A Combined CLSM and SEM Approach. Biomacromolecules 2006;7(8) 2310-2320.

[39] Ishihara S, Nakauma M, Funami T, Nakaura Y, Inouchi N, Nishinari K. Functions of Gum Arabic and Soybean Soluble Polysaccharide in Cooked Rice as a Texture Modifier. Bioscience Biotechnology and Biochemistry 2010;74(1) 101-107.

[40] Trivedi D, Bennett R J, Hemar Y, Reid D C W, Lee S K, Illingworth D. Effect of Different Starches on Rheological and Microstructural Properties of (I) Model Processed Cheese. International Journal of Food Science \& Technology 2008a;43(12) 2191-2196.

[41] Trivedi D, Bennett R J, Hemar Y, Reid D C W, Lee S K, Illingworth D. Effect of Different Starches on Rheological and Microstructural Properties of (II) Commercial Processed Cheese. International Journal of Food Science \& Technology 2008b;43(12) 21972203.

[42] Straadt I K, Thybo A K, Bertram H C. NaCl-Induced Changes in Structure and Water Mobility in Potato Tissue as Determined by CLSM and LF-NMR. LWT - Food Science and Technology 2008;41(8) 1493-1500.

[43] Bordoloi A, Kaur L, Singh J. Parenchyma Cell Microstructure and Textural Characteristics of Raw and Cooked Potatoes. Food Chemistry 2012;133(4) 1092-1100.

[44] Do T A L, Vieira J, Hargreaves J M, Mitchell J R, Wolf B. Structural Characteristics of Cocoa Particles and Their Effect on the Viscosity of Reduced Fat Chocolate. LWT Food Science and Technology 2011;44(4) 1207-1211. 
[45] Svanberg L, Ahrné L, Lorén N, Windhab E. Effect of Sugar, Cocoa Particles and Lecithin on Cocoa Butter Crystallisation in Seeded and Non-Seeded Chocolate Model Systems. Journal of Food Engineering 2011a;104(1) 70-80.

[46] Svanberg L, Ahrné L, Lorén N, Windhab E. Effect of Pre-Crystallization Process and Solid Particle Addition on Microstructure in Chocolate Model Systems. Food Research International 2011b;44(5) 1339-1350.

[47] Ong L, Dagastine R R, Kentish S E, Gras S L. The Effect of pH at Renneting on the Microstructure, Composition and Texture of Cheddar Cheese. Food Research International 2012;48(1) 119-130.

[48] Costa N E, O'Callaghan D J, Mateo M J, Chaurin V, Castillo M, Hannon J A, McSweeney P L H, Beresford T P. Influence of an Exopolysaccharide Produced by a Starter on Milk Coagulation and Curd Syneresis. International Dairy Journal 2012;22(1) 48-57.

[49] Richoux R, Aubert L, Roset G, Briard-Bion V, Kerjean J R, Lopez C. Combined Temperature-Time Parameters During the Pressing of Curd as a Tool to Modulate the Oiling-Off of Swiss Cheese. Food Research International 2008;41(10) 1058-1064.

[50] Adedeji A A, Liu L, Ngadi M O. Microstructural Evaluation of Deep-Fat Fried Chicken Nugget Batter Coating Using Confocal Laser Scanning Microscopy. Journal of Food Engineering 2011;102(1) 49-57.

[51] Loveday S M, Hindmarsh J P, Creamer L K, Singh H. Physicochemical Changes in a Model Protein Bar During Storage. Food Research International 2009;42(7) 798-806. 